ARTICLE

Received 14 Mar 2016 | Accepted 1 Sep 2016 | Published 10 Oct 2016

DOl: 10.1038 /ncomms13095

OPEN

\title{
Identification of a common mesenchymal stromal progenitor for the adult haematopoietic niche
}

\author{
Xingbin Hü ${ }^{1,2}{ }^{\star}$, Mayra Garcia ${ }^{1, \star}$, Lihong Weng ${ }^{1}$, Xiaoman Jung ${ }^{1}$, Jodi L. Murakami ${ }^{1,3}$, Bijender Kumar ${ }^{1}$, \\ Charles D. Warden ${ }^{4}$, Ivan Todorov ${ }^{5} \&$ Ching-Cheng Chen ${ }^{1,3}$
}

Microenvironment cues received by haematopoietic stem cells (HSC) are important in regulating the choice between self-renewal and differentiation. On the basis of the differential expression of cell-surface markers, here we identify a mesenchymal stromal progenitor hierarchy, where $\mathrm{CD} 45^{-}$Ter119- $\mathrm{CD} 31^{-} \mathrm{CD} 166^{-} \mathrm{CD} 146^{-} \mathrm{Sca} 1^{+}\left(\mathrm{Sca} 1^{+}\right)$progenitors give rise to $\mathrm{CD}_{45}{ }^{-} \mathrm{Ter}_{119}{ }^{-} \mathrm{CD} 31^{-} \mathrm{CD}^{-} 66^{-} \mathrm{CD}^{-} 46^{+}\left(\mathrm{CD} 146^{+}\right)$intermediate and $\mathrm{CD} 45^{-}$ Ter119- ${ }^{-}$D $31^{-} \mathrm{CD}_{166}{ }^{+} \mathrm{CD}_{146}{ }^{-}\left(\mathrm{CD}_{166}{ }^{+}\right)$mature osteo-progenitors. All three progenitors preserve HSC long-term multi-lineage reconstitution capability in vitro; however, their in vivo fates are different. Post-transplantation, $\mathrm{CD}_{146}{ }^{+}$and $\mathrm{CD} 166^{+}$progenitors form bone only.

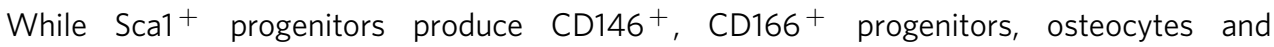
CXCL12-producing stromal cells. Only Sca1 ${ }^{+}$progenitors are capable of homing back to the marrow post-intravenous infusion. Ablation of $\mathrm{Sca}^{+}{ }^{+}$progenitors results in a decrease of all three progenitor populations as well as haematopoietic stem/progenitor cells. Moreover, suppressing production of KIT-ligand in $\mathrm{Scal}^{+}$progenitors inhibits their ability to support HSCs. Our results indicate that Sca1 ${ }^{+}$progenitors, through the generation of both osteogenic and stromal cells, provide a supportive environment for hematopoiesis.

\footnotetext{
${ }^{1}$ Divison of Hematopoietic Stem Cell and Leukemia Research, Gehr Family Center for Leukemia Research, Beckman Research Institute of City of Hope, Duarte, California 91010, USA. ${ }^{2}$ Department of Transfusion Medicine, Xijing Hospital, Fourth Military Medical University, Xi'an 7100032, PR China. ${ }^{3}$ Irell \& Manella Graduate School of Biological Sciences, City of Hope, Duarte, California 91010, USA. ${ }^{4}$ Bioinformatics Core, Department of Molecular Medicine, Beckman Research Institute of City of Hope, Duarte, California 91010, USA. ${ }^{5}$ Department of Diabetes and Metabolic Diseases Research, Beckman Research Institute of City of Hope, Duarte, California 91010, USA. * These authors contributed equally to this work. Correspondence and requests for materials should be addressed to C.-C.C. (email: chingchengchen@coh.org).
} 
$\mathrm{H}$ aematopoietic stem cells (HSCs) reside in highly specific bone marrow (BM) microenvironments (known as niches) that regulate their survival, proliferation and differentiation. Both intrinsic and extrinsic regulatory cues are integrated within the niche to maintain effective control over HSCs, ensuring they support hematopoiesis without inducing aberrant proliferation ${ }^{1-3}$.

Many studies have investigated the cellular compositions and anatomical site(s) of hematopoietic niches. Osteoblasts, endothelial cells, adipocytes and several variants of perivascular stromal cells including the CD146-expressing cells in humans, nestin ${ }^{+}$mesenchymal stromal cells (MSCs), leptin receptor-expressing mesenchymal cells, $\mathrm{Mxl}^{+}$stromal cells and CXCL12-abundant reticular (CAR) cells have all been proposed to participate in the regulation of HSCs in the $\mathrm{BM}^{4}$.

MSCs are currently defined as a cell population with colony forming capability (colony forming unit-fibroblastic, CFU-F) and the ability to undergo osteogenic, chondrogenic and adipogenic differentiation in vitro ${ }^{5,6}$. An obstacle to understanding the role of MSCs in the HSC niche is the heterogeneous nature of the MSC population and the need to identify functionally distinct subgroups. Studies have addressed this problem by identifying markers such as Osterix, LepR, Nestin, Sca1, CD146 and CD166 that can distinguish MSC subtypes $^{4}$, but a functional hierarchy that defines a lineage of early and late progenitors has not been identified. Therefore, it is difficult to assess the relationships within the niche cell population as well as the individual contributions of different cell types to support HSCs.

We have established an in vivo ectopic bone-forming assay in which the cellular and molecular components of the HSC niche can be genetically modified and explored. In this system, fetal bone cells are introduced under the kidney capsule, a highly vascularized region known to support tissue engraftments. Using this assay, we identified a fetal osteochondral progenitor as the HSC niche-initiating cell ${ }^{7}$. A recent fate-mapping study showed that the fetal niche-initiating cells and adult niche maintenance cells are distinct; they found that $\mathrm{LepR}^{+}$ mesenchymal stromal cells arise postnatally and give rise to bone and adipocyte cells in the adult bone marrow ${ }^{8}$. Here, we identify markers that can subdivide the mesenchymal stromal cell population into early and late progenitors that are functionally distinct. Using the in vivo ectopic bone-forming assay, we identified a mesenchymal stromal progenitor hierarchy in the BM: CD45 ${ }^{-}$Ter119 ${ }^{-} \mathrm{CD} 31^{-} \mathrm{CD} 166^{-} \mathrm{CD} 146^{-}$ $\mathrm{Scal}^{+}\left(\mathrm{Scal}^{+}\right)$cells are the most primitive, giving rise to intermediate progenitors $\mathrm{CD} 45^{-}$Ter119 ${ }^{-} \mathrm{CD} 31^{-} \mathrm{CD} 166^{-}$ $\mathrm{CD}_{146}{ }^{+}\left(\mathrm{CD} 146^{+}\right)$and mature osteo-progenitors $\mathrm{CD}_{4}{ }^{-}$Ter1 $19^{-} \mathrm{CD}^{-} 1^{-} \mathrm{CD}_{166^{+}} \mathrm{CD}_{146^{-}} \quad\left(\mathrm{CD} 166^{+}\right)$. All three progenitors display the characteristics of mesenchymal stromal cells and posses the ability to support hematopoiesis in vitro. Their differentiation potential and ability to traffic back to the $\mathrm{BM}$ in vivo varies. $\mathrm{CD} 146^{+}$and $\mathrm{CD} 166^{+}$progenitors form only bone in vivo; in contrast, $\mathrm{Scal}^{+}$cells differentiate into CXCL12-producing stromal cells, $\mathrm{CD} 146^{+}$intermediate mesenchymal progenitors and $\mathrm{CD} 166^{+}$mature osteoprogenitors. Ablation of $\mathrm{Sca}^{+}$cells results in a decrease of $\mathrm{CD}_{146}{ }^{+}$and $\mathrm{CD}_{166}{ }^{+}$cells, supporting our hierarchical designation; as well as a reduction in Lineage $^{-} \mathrm{Scal}^{+}$ ckit $^{+}$(LSK) cells and long-term HSCs (LT-HSCs; LSK$\left.\mathrm{CD} 150^{+} \mathrm{CD} 48^{-} \mathrm{CD} 135^{-}\right)$. Using shRNA knockdown, we show that KIT-ligand (KITL) gene expression in $\mathrm{Sca}^{+}$ progenitor derived cells is indispensable for maintaining HSCs. Our findings suggest that $\mathrm{Sca}^{+}$progenitors act as a common progenitor of mesenchymal stromal cells in the adult niche.

\section{Results}

Identification of three mesenchymal stromal progenitors. Although fetal $\mathrm{CD}_{105^{+}}$Thy1.1 ${ }^{-}$osteochondral progenitors formed ectopic bone with marrow under the kidney capsule ${ }^{7}$, the phenotypically similar adult $\mathrm{CD} 105^{+}$Thy $1.1^{-}$populations formed only bone without marrow after transplantation (Supplementary Fig. 1a,b). This result suggests that adult stromal progenitors may have different developmental potentials for niche formation and/or different surface markers compared with their fetal counterparts, therefore further analysis is needed to find an adult common progenitor. We subdivided the stromal population by first separating mouse marrow from crushed bone. A large portion of the HSCs and their supporting niche cells reside in the trabecular bone region ${ }^{9,10}$. To isolate the supporting niche cells, it is necessary to disassociate them from crushed bone using collagenase digestion. We designated these cells as the bone-disassociated stroma fraction, which may be part of either or both the endosteal and the perivascular niches. We then removed haematopoietic and endothelial cells by selecting

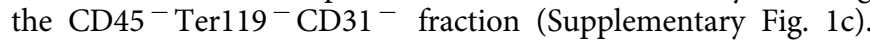
We next subdivided this population based upon cell-surface expression of CD146, a marker for human osteoprogenitors ${ }^{11}$, CD166, an adhesion molecule expressed by both HSCs and MSCs $^{12}$, and Sca1, a marker expressed by haematopoietic progenitors. Flow cytometry analysis revealed that the distributions of $\mathrm{CD}_{146}{ }^{+}$and $\mathrm{CD} 166^{+}$stromal cells were similar between bone-disassociated stroma and marrow fractions (Fig. 1a). However, the frequency of Scal ${ }^{+}$cells was noticeably lower in the marrow compartment, $0.004 \pm 0.00002 \%$ versus $0.056 \pm 0.007 \%$ (Fig. 1a and Supplementary Fig. 1d). Furthermore, most of the $\mathrm{Scal}^{+}$cells were located at the epiphysis, not diaphysis (Supplementary Fig. 1e,f). To determine the colony forming potential of each stromal subset, single stromal cells were sorted and cultured for 14 days. We observed colony forming activities, from bone-disassociated cells but not marrow-derived cells (Fig. 1b). Although the $\mathrm{Sca}^{+}$cells had the highest colony forming ability, cultured Scal ${ }^{+}, \mathrm{CD}_{146^{+}}$ and $\mathrm{CD} 66^{+}$cells all demonstrated slightly different, but very typical MSCs morphology (Supplementary Fig. 1g). The Sca1 ${ }^{-}$ population while more abundant $(0.388 \pm 0.058 \%$ versus $0.056 \pm 0.007 \%)$ had lower colony forming ability compared with $\mathrm{Scal}^{+}$cells (Fig. 1b).

Immunofluorescence staining of femur cross sections using Scal antibodies revealed that most $\mathrm{Sca}{ }^{+}{ }^{+}$cells are localized close to the endosteal surface in the trabecular bone region, especially in the lower part of the epiphyseal plate (Fig. 1c). Staining for Sca1 in Flk1-GFP transgenic mice, in which all the endothelial cells express GFP, revealed that many $\mathrm{Sca}^{+}{ }^{+}$stromal cells were located adjacent to the vasculature within the trabecular bone region (Fig. 1c,d). Three-dimensional visualization of confocal $z$-stacks illustrated that $\mathrm{Sca}{ }^{+}{ }^{+}$and $\mathrm{CD}_{146^{+}}$stromal cells, while closely associated with endothelial cells, were distinct cells (Fig. 1d) white arrows point to stromal cells, yellow arrows point to endothelial cells. To distinguish $\mathrm{Sca}{ }^{+}{ }^{+} \mathrm{HSC}$ from Sca1 ${ }^{+}$ stromal cells, haematopoietic cells were stained with an antibody against CD45, this highlighted a distinct population of $\mathrm{CD}_{4} 5^{-} \mathrm{Sca}{ }^{+}$perivascular cells that were wrapped around the endothelial cells (Fig. 1d). Co-staining with laminin, a protein abundant in basement membranes that encases the BM sinusoidal vessels and perivascular cells ${ }^{13}$ confirmed that Sca1 ${ }^{+}$ cells are largely localized in the perivascular region (Fig. 1e). $\mathrm{CD}_{146}{ }^{+}$cells were also located in the perivascular region and had similar distribution as Sca1 ${ }^{+}$cells (Fig. 1d,e). CD166 ${ }^{+}$cells, unlike Sca1 ${ }^{+}$and $\mathrm{CD}_{146}{ }^{+}$cells, were mainly localized to the endosteal surface and not associated with the vasculature (Supplementary Fig. 1h). 
a

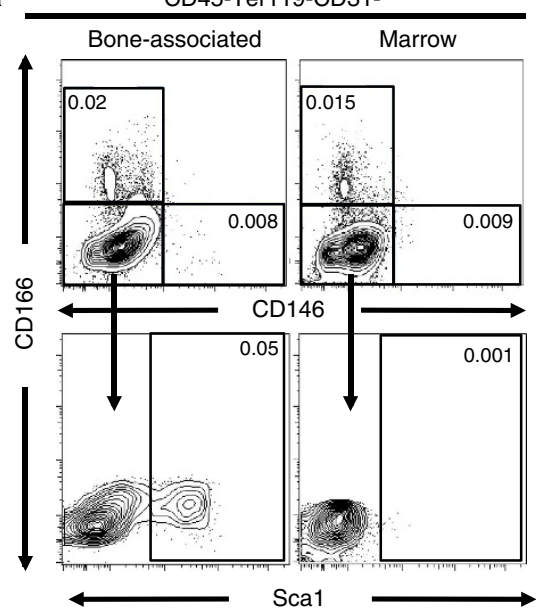

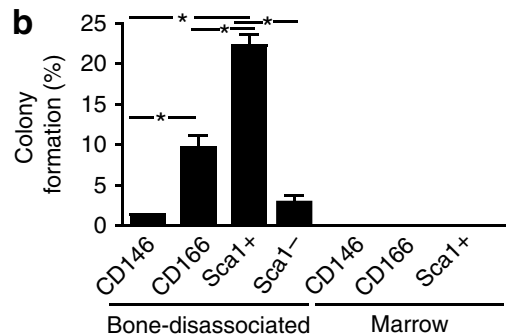

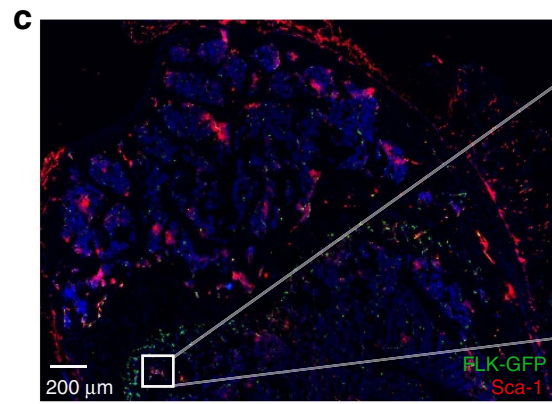

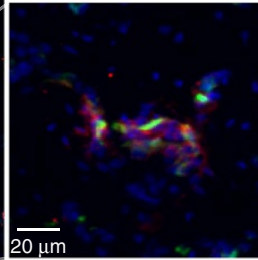

$20 \mu \mathrm{m}$

d
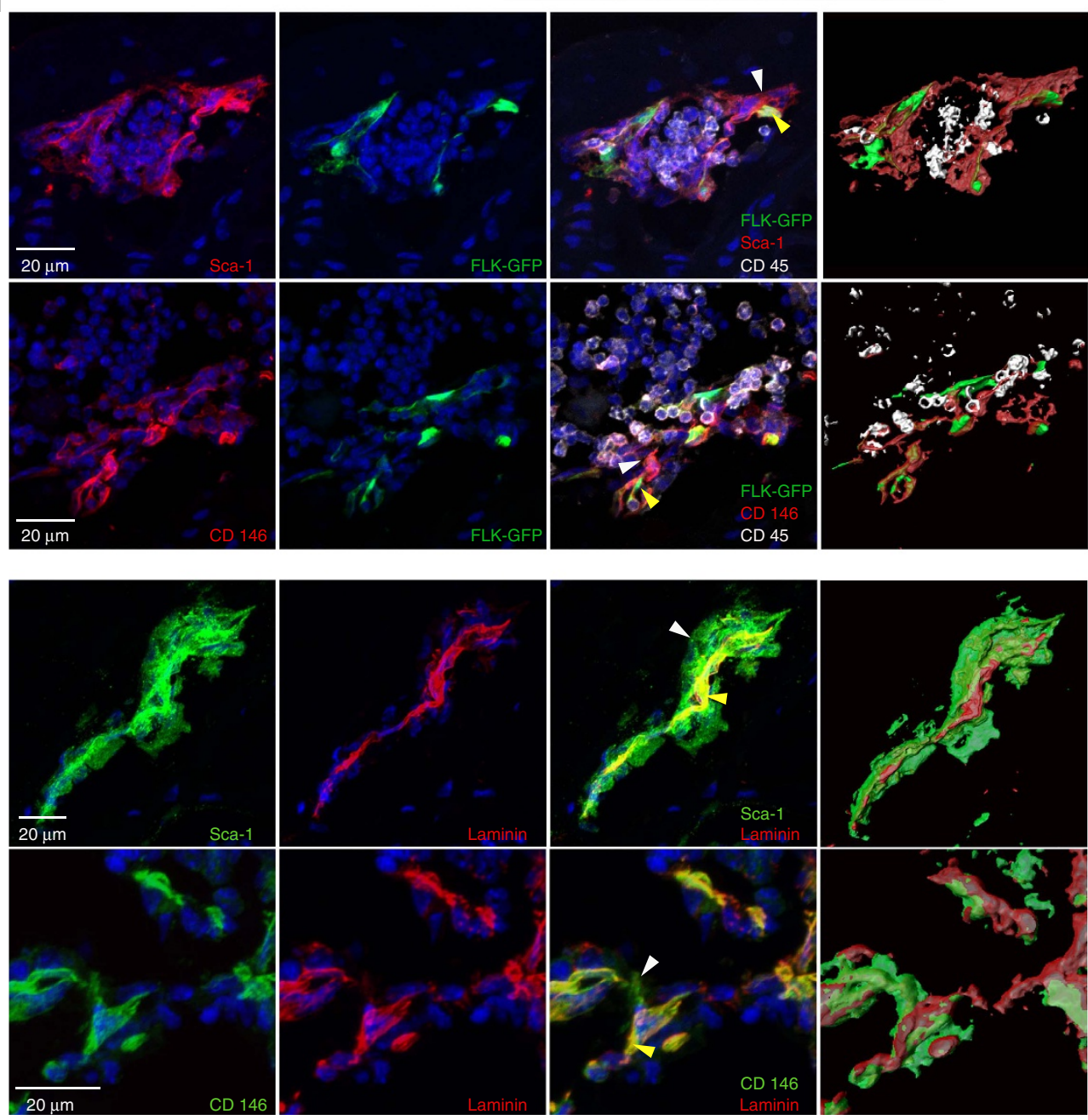

Figure 1 | Phenotypic Identification of three bone-disassociated mesenchymal progenitors. (a) Representative FACS profiles of stromal cells $\left(C D 45^{-}\right.$Ter119 ${ }^{-} \mathrm{CD}^{-} 1^{-}$) near the endosteum (bone-disassociated). They were separated based on their CD166, CD146 and Sca1 expression profiles. Numbers shown in the gate represent the percentage of total live cells. (b) Single-cell colony forming efficiency of BM stromal progenitors ( ${ }^{*} P \leq 0.05$, three repeats of 60 cells each, student's t-test). (c) Sections of femur from Flk1-GFP mouse stained with antibodies against Sca1 and scanned with an iCys Research imaging cytometer. Nuclei were stained with Dapi. (d) Confocal maximum intensity projections of sections of femurs from Flk1-GFP mice stained with antibodies against Sca1 or CD146; scanned near the epiphyseal plate. (e) Confocal maximum intensity projections of sections of femur stained with antibodies against Sca1 or CD146 and laminin; scanned near epiphyseal plate. White arrows point to mesenchymal progenitors yellow arrows point to endothelial cells. 
$\mathrm{Scal}^{+}$progenitors contribute to $\mathrm{BM}$ stroma formation. To determine if the adult mesenchymal progenitors possessed similar niche-initiating capabilities to the fetal osteochondral progenitors, we transplanted bone-disassociated $\mathrm{CD} 146^{+}, \mathrm{CD}_{166^{+}}$or Scal ${ }^{+}$ progenitors under the mouse kidney capsule (Fig. 2a). One month later we observed no marrow formation from these grafts. The $\mathrm{CD} 146^{+}$and $\mathrm{CD} 166^{+}$progenitors produced bone, whereas the $\mathrm{Sca}{ }^{+}$cells produced a limited fibroblast-like cell growth (Fig. 2b). This suggests that $\mathrm{CD}_{146}{ }^{+}$and $\mathrm{CD} 166^{+}$cells are likely osteo-progenitors committed to an osteoblastic fate, while $\mathrm{Sca}^{+}$cells may be more primitive, and require signals not present in the kidney capsule in order to differentiate into a specific cell type. Moreover, although all three stromal subsets have classically defined MSC characteristics, the $\mathrm{Sca}{ }^{+}$cells can be distinguished by their in vivo differentiation potential.

The in vivo relationship with other niche cells can potentially alter stromal cell differentiation. To model the multiple cell populations in the developing niche we co-transplanted GFP-expressing bone-disassociated adult progenitors, isolated from C57BL/Ka-Thy1.1-CD45.1-GFP mice, with unmarked fetal skeletal progenitors under the kidney capsule (Fig. 2c). Progeny of $\mathrm{CD}_{146}{ }^{+}$and $\mathrm{CD} 166^{+}$progenitors could only be found in the bone portion of the graft, and not in the marrow area of the graft (Supplementary Fig. 2a,b). The Sca1 ${ }^{-}$cells failed to contribute to the graft evidenced by the lack of $\mathrm{GFP}^{+}$cells (Supplementary Fig. 2c). In contrast, $\mathrm{Sca}^{+}$progenitor derived cells could clearly be identified in the area beneath the bone (Fig. 2d). A cross-section of the graft revealed that donor-derived GFP ${ }^{+}$cells mainly localized within the marrow compartment and had a reticular cell-like structure, with some cells encircling the vasculature (Fig. 2d and Supplementary Fig. 2g). Staining with anti-GFP antibody confirmed that the Scal ${ }^{+}$-derived cells were located within the marrow portion of the bone graft (Supplementary Fig. 2d). Flourescent-activated cell sorting (FACS) analysis of the kidney graft indicated that $\mathrm{Sca}^{+}$ progenitors gave rise to phenotypic CAR cells $(61.67 \pm .37 .53 \%)$ $\left[\mathrm{Sca}{ }^{-} \mathrm{CD} 44^{+} \mathrm{CD} 51^{+} \mathrm{CD} 106^{+} \mathrm{CD} 140 \mathrm{a}^{+}\right.$(ref. 14)] and Sca1 ${ }^{+}$ stromal cells in the marrow of the graft (Fig. 2e-g). Donorderived $\mathrm{CD}_{146}{ }^{+}(75.00 \pm 30.32 \%), \mathrm{CD}{ }^{+}(79.13 \pm 6.976 \%)$ and $\mathrm{Scal}^{+}(90.07 \pm 10.52 \%)$ cells were found in the bonedisassociated fraction of the graft (Fig. 2e,g). The kidney cells are highly auto-fluorescent thus we stained kidney cells and used them as a negative control to set the GFP gate (Fig. 2e). When we used a more stringent gate for GFP Scal ${ }^{+}$-derived $\mathrm{CD} 146^{+}$, $\mathrm{CD} 66^{+}{ }^{+}$and Scal ${ }^{+}$were still identified (Supplementary Fig. 2e). We found neither haematopoietic $\left(\mathrm{CD} 45^{+}\right)$nor endothelial $\left(\mathrm{CD} 31^{+}\right)$contributions from the transplanted $\mathrm{GFP}^{+}$cells by immunostaining of graft sections, suggesting that the $\mathrm{Sca}{ }^{+}$ $\mathrm{GFP}^{+}$transplanted cells were a pure population (Supplementary Fig. 2f,g). Furthermore, we show by FACS analysis that the $\mathrm{CD} 45^{+}$cells found in the kidney transplanted with $\mathrm{Sca}{ }^{+} \mathrm{GFP}^{+}$ cells are negative for GFP and the CD45 ${ }^{-}$Ter $119^{-} \mathrm{CD} 31^{-}$cells are positive for GFP (Supplementary Fig. 3a,b). We sorted CD45 ${ }^{-}$Ter119 ${ }^{-} \mathrm{CD}^{-} 1^{-}$GFP low and GFP high cells from a kidney transplanted with $\mathrm{Sca}^{+}{ }^{+} \mathrm{GFP}^{+}$cells and found that they expressed GFP by quantitative real-time PCR (qRT-PCR), while cells from a control untransplanted kidney did not (Fig. 2h). Furthermore, the control cells, which were mixed population of stromal, haematopoietic and endothelial cells, had high expression of CD45 and CD31. The sorted GFP ${ }^{+}$cells, in contrast, had undetectable levels of CD45 and CD31 suggesting that the transplanted cells are not derived from and do not give rise to haematopoietic or endothelial cells (Fig. 2h). Similar results were obtained after cells from a clone of a single Sca1 ${ }^{+}$progenitor were transplanted with fetal cells or alone under the renal capsule, this confirmed that the various cell types were derived from a single progenitor and not from a mixed population within the $\mathrm{Scal}^{+}$sorted progenitors (Supplementary Fig. 3c,d). These results support a view where $\mathrm{Scal}^{+}$cells are primitive mesenchymal stromal progenitors, while CD146 ${ }^{+}$and $\mathrm{CD} 166^{+}$ are more mature osteo-progenitors as they were only found to give rise to bone cells.

The fact that $\mathrm{CD}_{146}{ }^{+}$and $\mathrm{CD} 66^{+}$stromal subsets were only found in the bone portion of the graft suggests they mainly contain osteoblast progenitors. This is further supported by our analysis of BM stromal cells in Osx1-Cre:GFP and Col2.3-GFP transgenic mice, in which osteo-progenitors and mature osteoblasts, respectively, are marked by GFP expression $^{15,16}$. In Col2.3-GFP mice, more bone-disassociated $\mathrm{CD} 166^{+}(27.83 \pm 1.63 \%)$ cells than $\mathrm{CD}^{+}{ }^{+}(1.91 \pm 0.72 \%)$, $\mathrm{Scal}^{+}(3.55 \pm 1.18 \%)$ and $\mathrm{Scal}^{-}(1.82 \pm 0.68)$ expressed GFP (Fig. 3a). Because the $\mathrm{Scal}^{-}$population is more abundant the majority of GFP-expressing cells were Scal ${ }^{-}$(Fig. 3c). In the Osx1-Cre;GFP mice the majority of GFP-expressing cells were also Sca ${ }^{-}$(Fig. 3d), although the frequency of GFP was hig her in the $\mathrm{CD} 166^{+}$population than in the $\mathrm{Sca}{ }^{-}$population $(4.01 \pm 0.27 \%$ versus $0.41 \pm 0.005 \%)$ (Fig. $3 b)$. This suggests that $\mathrm{CD} 66^{+}$cells are more enriched for osteo-progenitors and osteoblast. Immunofluorescence staining revealed that most $\mathrm{Sca}^{+}$cells did not co-localize with the $\mathrm{GFP}^{+}$cells in these animals (Fig. 3e,f). All together, the results suggest that $\mathrm{CD} 146^{+}$, $\mathrm{CD} 66^{+}$and $\mathrm{Scal}^{+}$progenitors function differently in vivo. $\mathrm{Sca}^{+}{ }^{+}$cells were the most primitive of all three progenitors; they preserved themselves after in vivo transplantation and gave rise to multiple cell types (Fig. 2e-g), and had a high cloning efficiency (Fig. 1b), while $\mathrm{CD} 146^{+}$and $\mathrm{CD} 166^{+}$appear to be osteo-progenitors.

Only Scal ${ }^{+}$progenitors home to $\mathrm{BM}$ after intravenous infusion. We next tested the physiological role of bone-disassociated mesenchymal progenitors in their endogenous BM microenvironment. To evaluate the homing ability of $\mathrm{CD}_{146}{ }^{+}, \mathrm{CD}_{166}{ }^{+}$, $\mathrm{Scal}^{-}$and $\mathrm{Scal}^{+}$progenitors, we injected freshly isolated GFPexpressing progenitors from C57BL/Ka-Thy1.1-CD45.1-Tg (CAG-GFP) mice intravenously into sub-lethally irradiated mice (Fig. 4a). One month later, we identified donor-derived stromal cells only in the $\mathrm{BM}$ of animals that received $\mathrm{Scal}^{+}$progenitors (Fig. 4b,c). No donor-derived tissue could be found in animals that received $\mathrm{PBS}, \mathrm{CD}_{146}{ }^{+}, \mathrm{CD}_{166}{ }^{+}$or $\mathrm{Sca}{ }^{-}$cells, by both immunohistochemistry and FACS analysis (Supplementary Fig. 4a). FACS analysis revealed that intravenously transferred $\mathrm{Sca}^{+}$progenitors gave rise to cells with surface markers of CAR $(9.43 \pm 8.558 \%)$ cells in the marrow fraction and $\mathrm{CD}_{146}{ }^{+}(4.99 \pm 3.993 \%), \mathrm{CD} 166^{+}(6.11 \pm 1.959 \%)$ and $\mathrm{Sca}{ }^{+}$ $(4.34 \pm 1.507 \%)$ cells in the bone-disassociated fraction (Fig. $4 \mathrm{~d}-\mathrm{f})$. To exclude the possibility that the $\mathrm{GFP}^{+}$cells observed in the $\mathrm{Scal}^{+}$IV transplanted mice were derived from HSCs, we stained sections with anti-CD45 and CD41 antibodies to show that GFP did not overlap with these markers (Supplementary Fig. 4b). FACS analysis also revealed that $\mathrm{CD} 45^{+}$and $\mathrm{CD} 31^{+}$cells in the $\mathrm{Scal}^{+}$IV transplanted mice do not express GFP, while $\mathrm{CD}^{-} 5^{-}$Ter119- CD31 ${ }^{-}$cells do (Supplementary Fig. 4c). qRT-PCR on the sorted bone-dissociated CD45- Ter119 ${ }^{-}$ $\mathrm{CD}^{-} 1^{-} \mathrm{GFP}^{+}$and $\mathrm{GFP}^{-}$cells isolated 1 month post IV-injection verified the GFP expression and showed that while the GFP ${ }^{-}$cells expressed CD45 and CD31, the $\mathrm{GFP}^{+}$cells did not, excluding the possibility that $\mathrm{GFP}^{+}$cells are derived from haematopoietic or endothelial lineages (Fig. 4g). Moreover the stromal nature of the $\mathrm{GFP}+$ cells is illustrated in the hematoxylin and eosin (H\&E) stained section (Fig. 4b, blue arrows). 
a

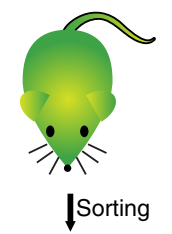

BM

stromal progenitors

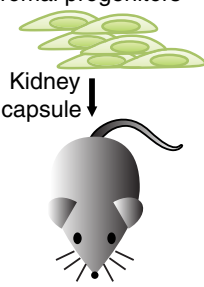

b

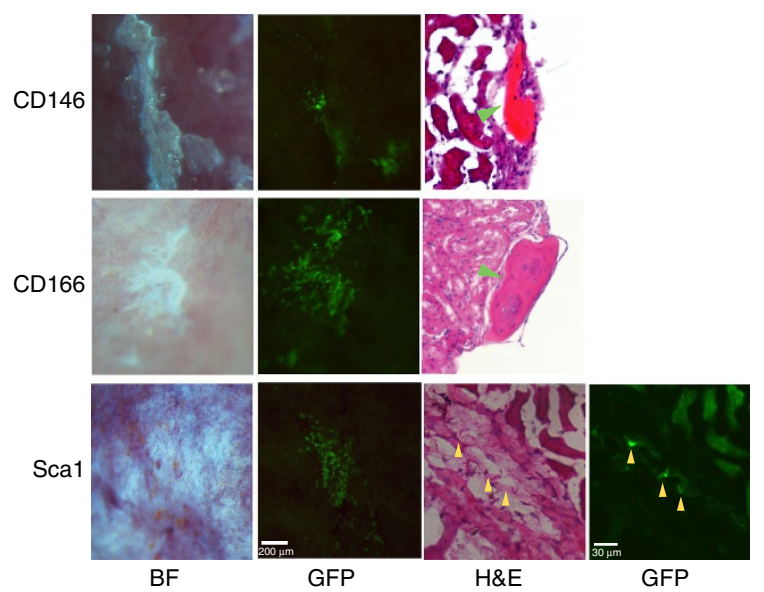

c

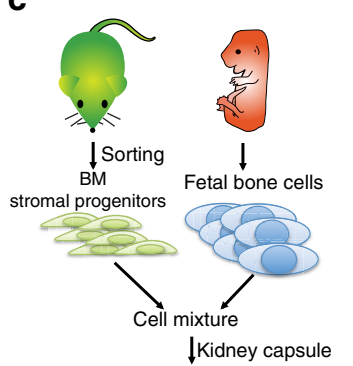

d

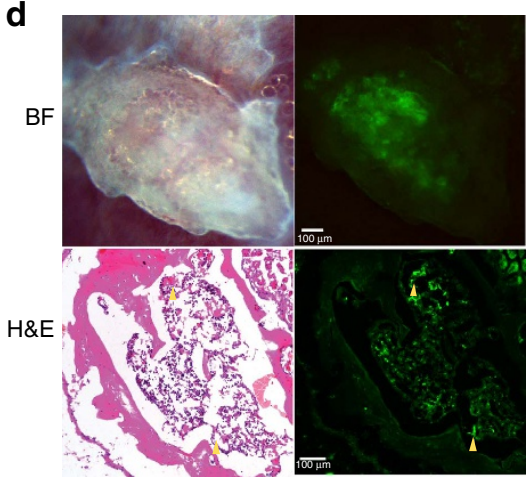

e

CD45-Ter119-CD31-

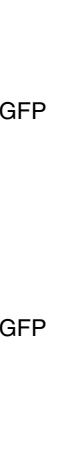

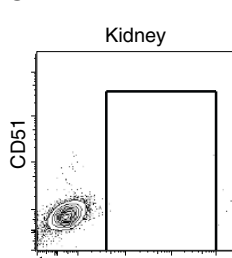

f

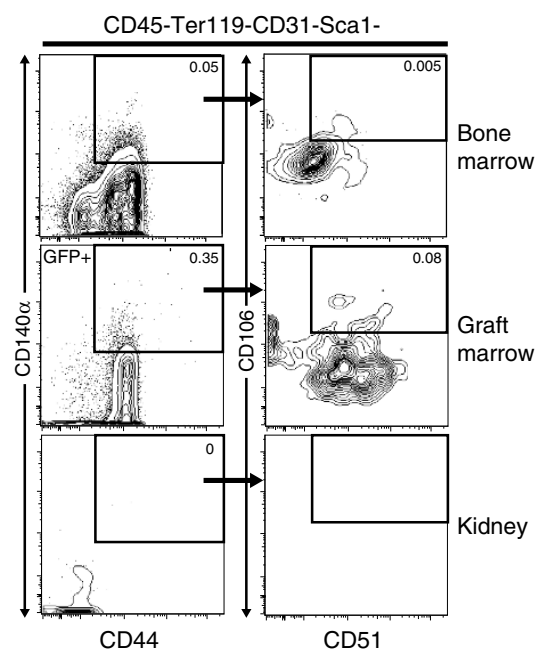

g

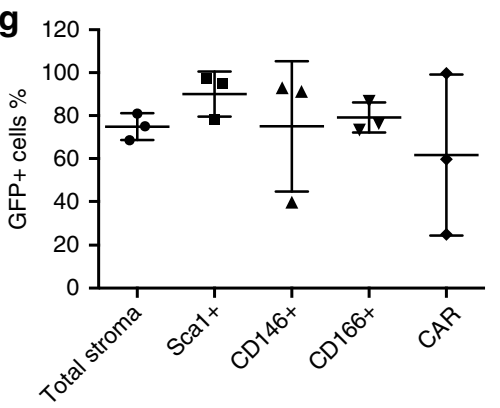

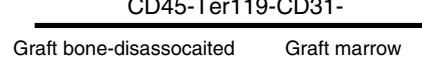

Graft bone-disassocaited Graft marrow

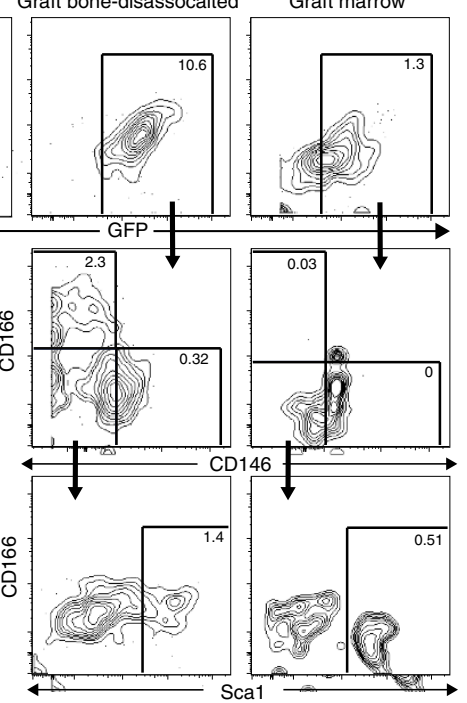

h
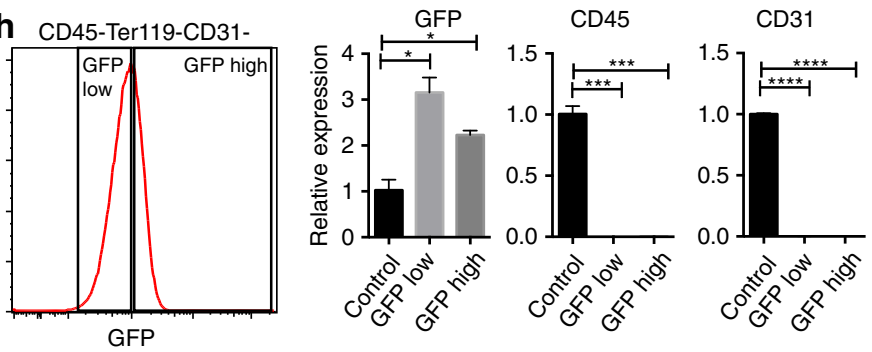

Figure 2 | Sca1 ${ }^{+}$progenitors contribute to BM stroma, while CD146 ${ }^{+}$and CD166 ${ }^{+}$progenitors form bones. (a) Direct transplants of GFP- labelled progenitors under the kidney capsule. (b) Bright-field and GFP images of GFP-labelled progenitors 1 month after transplant (far left and left). A representative cross-section of the graft site was stained with H\&E (right, green arrowhead points to bone) or GFP to identify the donor origin (far right, yellow arrowheads). (c) Co-tranplants of GFP-labelled adult progenitors with non-GFP fetal skeletal progenitors under the kidney capsule. (d) Bright-field and GFP images of GFP-labelled Sca1 ${ }^{+}$mixed with fetal skeletal progenitors 1 month after transplant. Donor-derived GFP ${ }^{+}$cells can be clearly identified (far left and left). Representative cross sections of the graft site stained with H\&E (right) or GFP ( far right) to identify the donor origin (yellow arrowheads). (e-f) Representative FACS analysis of graft of mixed GFP-labelled Sca1 ${ }^{+}$progenitors and non-GFP skeletal progenitors harvested 1 month after transplant. The per cent of live cells is displayed for each gate (e) FACS analysis of donor-derived endosteum associated progenitors (left) and marrow stromal cells (right). (f) FACS analysis for phenotypically defined CAR cells in control bone marrow, marrow of graft and kidney. (g) Percentage of GFP + cells for each group; mean \pm s.d. $(n=3)$. Total stroma is the Ter119-CD45-CD31- population. (h) Histrogram for GFP to show the populations that were sorted for RNA isolation (far left). Relative expression of GFP, CD45 and CD31 in control cells (untransplanted kidney), GFP low gate cells and GFP high gate cells $\left({ }^{\star} P<0.05,{ }^{\star \star \star} P<0.0005,{ }^{\star \star \star \star} P<0.00005\right.$, mean \pm s.d., technical triplicate, student's $t$-test. 
a CD45-TER119-CD31-

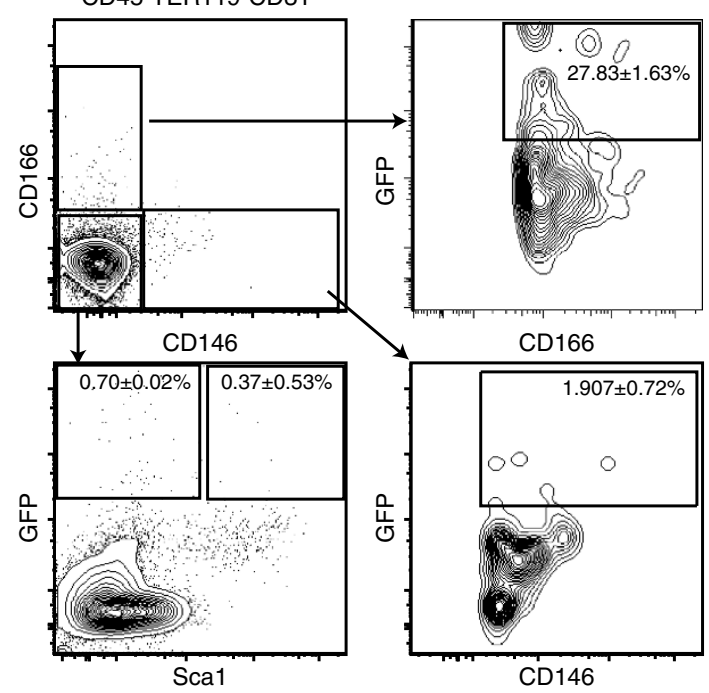

C

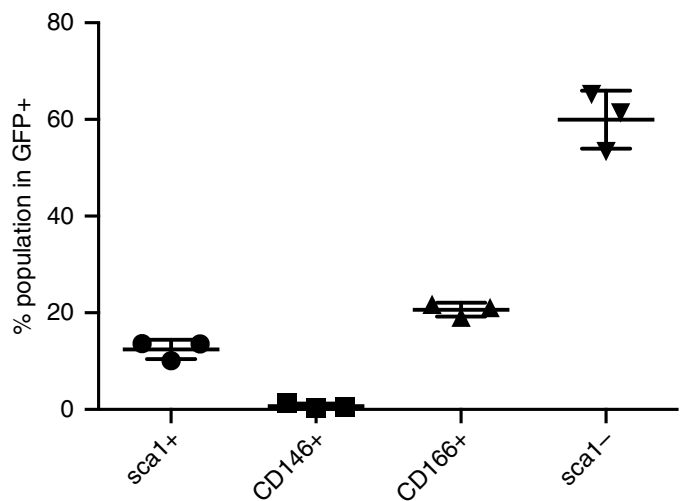

e

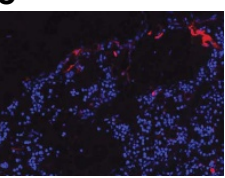

Sca1

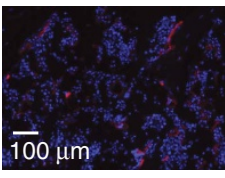

CD146

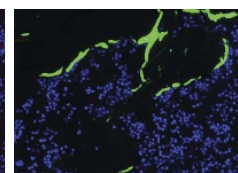

Col2.3-GFP

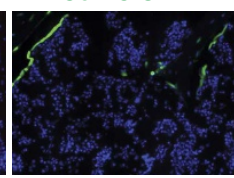

Col2.3-GFP

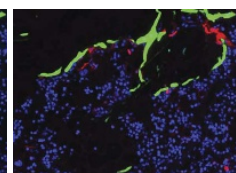

Merge

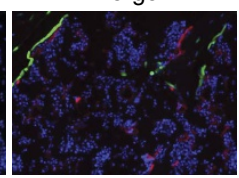

Merge

b

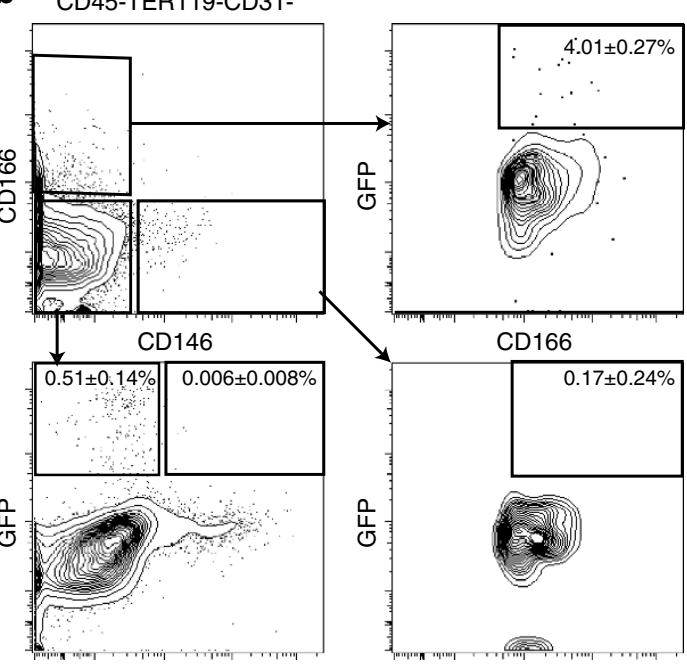

Sca1

CD146

d

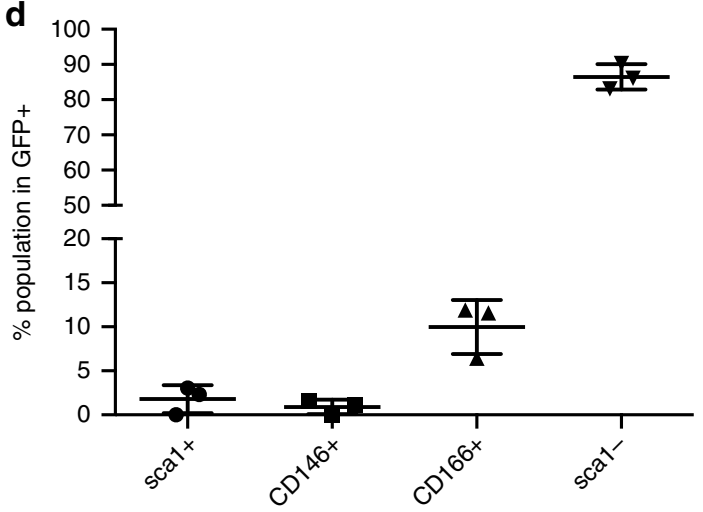

f

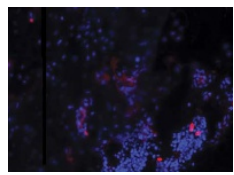

Sca1

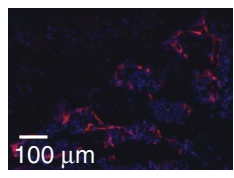

CD146

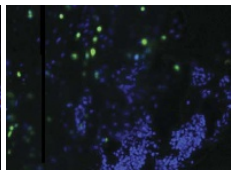

Osterix-GFP

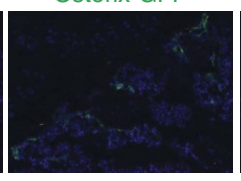

Osterix-GFP

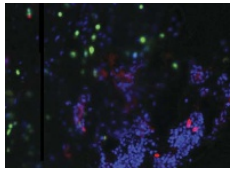

Merge

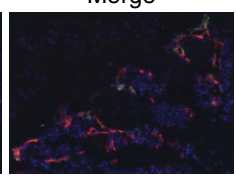

Merge

Figure 3 | CD $146^{+}$and CD $166^{+}$cells were marked by Col2.3-GFP or Osx1-Cre:GFP while Sca1 ${ }^{+}$cells were not. (a-b) FACS analysis of bonedisassociated stromal cells (CD45 ${ }^{-}$TER119 ${ }^{-}{\text {CD } 31^{-}}^{-}$) from (a) Col2.3-GFP or (b) Osx1-Cre:GFP mice. The fequency of the parent gate is displayed. (c-d) The frequency of Sca1 +, CD146 +, CD166 + and Sca1- in the GFP + population for (mean \pm s.d.) (c) Col2.3-GFP and (d) Osx1-Cre:GFP obtained from FACS analysis $(n=3)$. (e-f) Sections of femurs from (e) Col2.3-GFP or (f) Osx1-Cre:GFP mice stained with antidodies to Sca1 or CD146.

One of the key defining markers for CAR cells is the expression of the chemokine CXCL12 (ref. 17). We performed qRT-PCR to determine the CXCL12 expression levels in $\mathrm{Sca}^{+}$progenitors and their progeny. Sca1 ${ }^{+}$progenitors expressed CXCL12 at a low level compared with marrow CAR cells. However, after transplantation, $\mathrm{Scal}^{+}$progenitors gave rise to progeny that expressed CXCL12 at comparable levels to CAR cells in marrow, both in the kidney graft and IV transplantation (Supplementary Fig. 5a). Furthermore, we could detect CXCL12-producing, phenotypic CAR cells even when $\mathrm{Scal}^{+}$ cells were transplanted under the kidney capsule without fetal cells, suggesting that differentiation of $\mathrm{Scal}^{+}$progenitors into CAR cells may be a default state whereas environmental signals are necessary for the osteogenic fate (Supplementary Fig. 5a,b). To exclude the possibility that the observed donor-derived cells were a result of transplanted $\mathrm{Sca}^{+}$ progenitors fused with host cells, we injected $\mathrm{GFP}^{+}$ $\mathrm{Sca}^{+}$cells into sub-lethally irradiated mice that constitutively express the tdTomato fluorescent protein (tdTomato). We did not detect any cells that co-expressed GFP and tdTomato. These data suggest that the donor contributions arose from the proliferation and differentiation of transplanted $\mathrm{Scal}^{+}$progenitors and not from fusion with host cells (Supplementary Fig. 5c). 
a

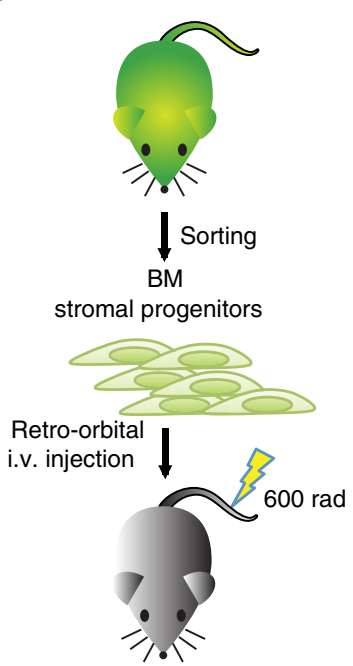

b

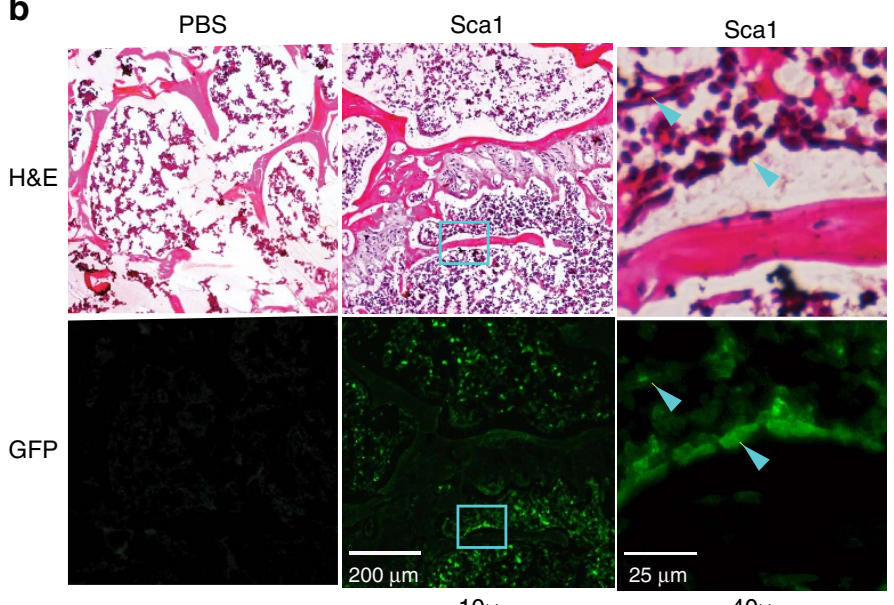

$10 \times$

$40 \times$ c

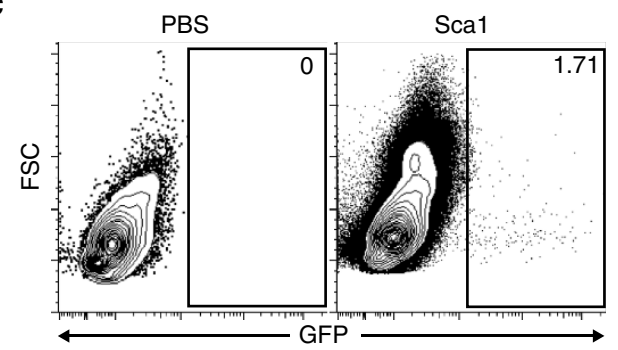

d

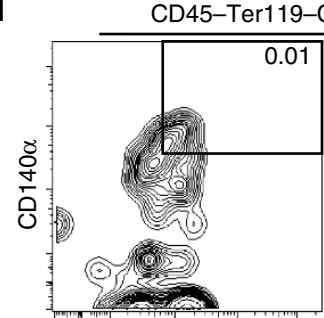

CD44

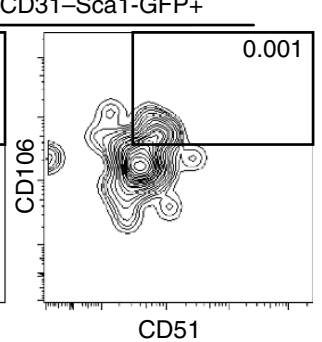

e
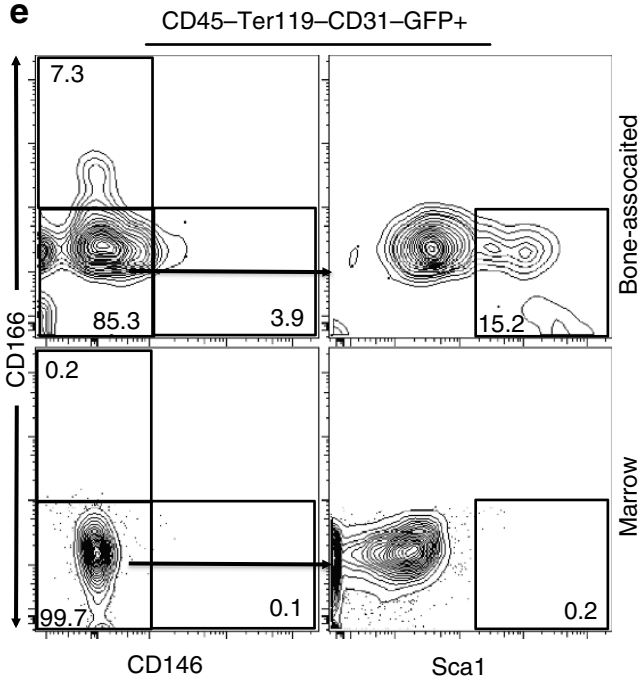

f

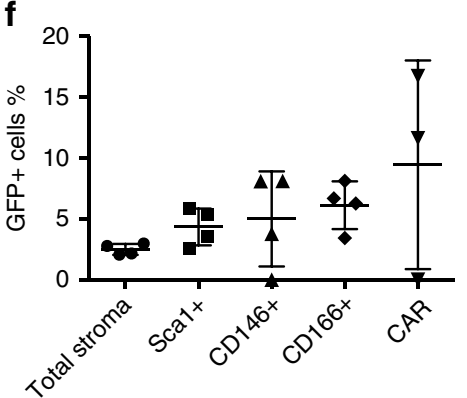

9 CD45-Ter119-CD31-
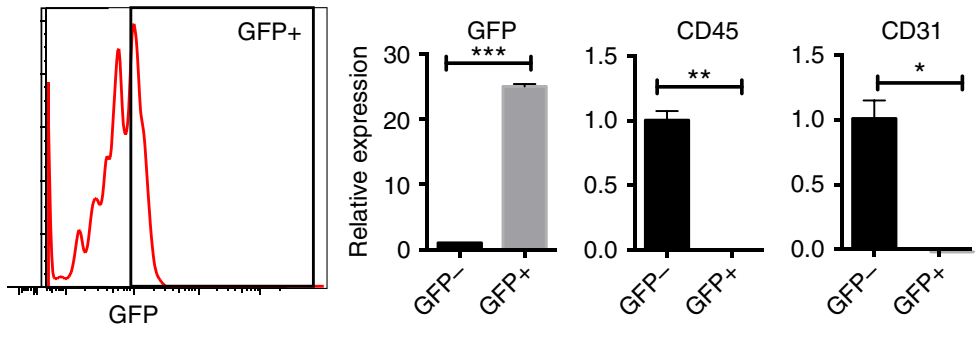

Figure 4 | Sca1 ${ }^{+}$progenitors hometo the BM after intravenous transplantation into irradiated mice. (a) Intravenous transfusion of GFP-lablled mesenchymal progenitors into irradiated mice $(600 \mathrm{rad})$. (b) Representative cross sections of the tibia stained with H\&E (upper) or GFP (lower). Tibias were harvested 1 month after transplantation. Blue arrows point to GFP + stromal cells (c-d) FACS analysis of the marrow of recipients injected with $\mathrm{Sca}^{+}$cells for (c) GFP expression or (d) phenotypes of CAR cells. (e) FACS analysis of donor-derived bone-disassociated progenitors (upper) and marrow stromal cells (lower). (f) Frequency of GFP + cells in each group; mean \pm s.d. $(n=4)$. (g) Histrogram of GFP to show the population that was sorted for RNA isolation (far left). Relative expression of GFP, CD45 and CD31 in GFP- and GFP + sorted cells from CD45- Ter119- CD31- stromal cell gate $\left({ }^{\star} P<0.05,{ }^{\star *} P<0.005,{ }^{\star \star \star} P<0.0005\right.$, mean \pm s.d., technical triplicate, student's $t$-test $)$.

Taken together, our results demonstrate that, among the three mesenchymal progenitors, only $\mathrm{Scal}^{+}$progenitors can home back to the $\mathrm{BM}$ and that they contribute to the formation of both bone and the BM microenvironment.
This result supports our conclusion that $\mathrm{Sca}^{+}$cells represent the most primitive progenitor and are capable of giving rise to $\mathrm{CD}_{146}{ }^{+}, \mathrm{CD} 166^{+}$cells and CAR cells. 
Mesenchymal progenitors express HSC supporting genes. To understand the molecular mechanisms underlying the functional differences among the $\mathrm{Scal}^{+}$mesenchymal stromal progenitor and $\mathrm{CD}_{146}{ }^{+}$and $\mathrm{CD}_{166}{ }^{+}$osteoprogenitors, we performed single-cell multi-gene qRT-PCR to measure the expression levels of 47 niche-related genes and the housekeeping gene, GAPDH. In agreement with our CFU-F studies, and despite the expression of similar surface markers, the bone-disassociated progenitors and marrow-localized cells had remarkably different gene expression profiles; the former expressed a wider repertoire of genes related to the support of HSC functions (Fig. 5a). On the basis of only the gene expression values, principal component analysis partitioned the bone-disassociated cells by their surface marker phenotype (Fig. 5b). Principal component 1, which captures the largest proportion of the variation in the data, separates the $\mathrm{Scal}^{+}$and $\mathrm{CD} 146^{+}$cells from the $\mathrm{CD} 166^{+}$cells. Principal component 2 further separates the $\mathrm{Scal}^{+}$and $\mathrm{CD}_{146}{ }^{+}$cells (Fig. 5b). However, although individual populations can be distinguished, there is also some overlap, particularly between the $\mathrm{Scal}^{+} / \mathrm{CD} 146^{+}$and $\mathrm{CD} 146^{+} / \mathrm{CD} 166^{+}$cells. These data suggest that $\mathrm{CD}_{146^{+}}$cells may be acting as an intermediary osteo-progenitor. We compared the gene expression values among the three bone-disassociated progenitors and identified statistically significant variations in expression levels of shared genes. Scal ${ }^{+}$progenitors expressed higher levels of cytokines that are important for skeletal development (BMP4, FGF2, FGF7 and IGF-1) and HSC quiescence (Angpt1). They also expressed cathepsin $\mathrm{K}$, an enzyme important for bone remodelling and absorption $^{18}$ (Fig. 5c). CD146 ${ }^{+}$cells expressed Angpt2, an antagonist of Angpt1, at higher levels than Sca1 ${ }^{+}$and $\mathrm{CD}_{166}{ }^{+}$ cells. Both $\mathrm{Scal}^{+}$and $\mathrm{CD}_{146^{+}}$cells expressed TIMP3, an endogenous metalloproteinase that can recruit quiescent HSCs into the cell cycle ${ }^{19}$, and KITL, a key player in maintaining HSCs, at higher levels than the $\mathrm{CD} 166^{+}$cells (Fig. 5d). Consistent with our immunofluorescence studies (Fig. 1d,e), bone-disassociated $\mathrm{Sca}^{+}$and $\mathrm{CD}_{146}{ }^{+}$cells also expressed high levels of CD140 $\beta$, a common marker for perivascular cells ${ }^{20}$ (Fig. $5 \mathrm{a}$,d). In agreement with our transgenic mouse (Fig. 3d) and transplantation studies (Fig. 2b), expression of osteocalcin, the mature osteoblast marker, was highest in bone-disassociated $\mathrm{CD} 166^{+}$cells and lower in $\mathrm{Sca}{ }^{+}$and $\mathrm{CD} 146^{+}$cells, suggesting $\mathrm{CD}^{+} 66^{+}$cells are more differentiated than both $\mathrm{Sca}^{+}$and $\mathrm{CD} 146^{+}$cells (Fig. 5a,e). $\mathrm{CD} 166^{+}$progenitors also expressed other osteo-lineage markers including Osterix and DKK1, a Wnt signalling inhibitor that suppresses bone formation ${ }^{21}$, at higher levels than $\mathrm{Scal}^{+}$and $\mathrm{CD} 146^{+}$cells (Fig. 5a,e). Taken together, these data support a model where the $\mathrm{CD}_{166}{ }^{+}$cells are the most mature and the $\mathrm{Scal}^{+}$progenitors are the most primitive with $\mathrm{CD} 146^{+}$possibly acting as an intermediary.

Nestin- and Leptin receptor-expressing mesenchymal stromal cells were previously shown to be important components of the HSC niche ${ }^{8,22,23}$. Interestingly, although nestin expression was identified in most of the bone-disassociated $\mathrm{CD} 46^{+}$and $\mathrm{CD} 66^{+}$cells, only a small portion of the Scal ${ }^{+}$ cells expressed nestin (Fig. 5b). Indeed, when we analysed the BM stromal cells in nestin-GFP transgenic mice, we found only a few cells in the marrow and bone-disassociated fractions that expressed both Scal and Nestin together (Supplementary Fig. 6a,b). Suggesting that Nestin expression may be a characteristic of more differentiated stromal cell types. Using the leptin receptor (LepR) as a maker, Sean Morrison's group identified a perivascular stromal population that is instrumental to HSC maintenance and can give rise to bone and adipose cells in the adult bone marrow ${ }^{8,23}$. We observed LepR antibody staining in $0.41 \pm 0.040 \%$ of live cells and $70.47 \pm 1.78 \%$ of the CD31-Ter119-CD45- stromal population (Supplementary Fig. 6c). The highest levels of LepR antibody staining were found in the $\mathrm{CD} 166^{+}(90.2 \pm 6.67 \%)$ subpopulation compared with Sca1 ${ }^{+}(74.9 \pm 5.86 \%), \mathrm{CD} 146^{+}(80.8 \pm 8.63 \%)$ and Sca1 ${ }^{-}$ $(66.73 \pm 2.20 \%)$ subpopulations (Supplementary Fig. 6d). Consistent with the antibody staining the mesenchymal stromal cell populations showed similar relative expression by qPCR, using a probe that recognizes all four transcripts of LepR (Supplementary Fig. 6e). Together these data suggest that Sca1, CD146 and CD166 could be used to subdivide the LepR-expressing mesenchymal cells, providing a more detailed view of the HSC niche.

Mesenchymal progenitors are important for HSC maintenance. To evaluate the individual ability of the identified bonedisassociated progenitors to support HSCs, we co-cultured sorted LT-HSCs with each progenitor. After two days, we plated the co-cultured HSCs as single cells per well in a cytokine cocktail that promotes full erythromyeloid potential. We then analysed individual wells by flow cytometry at day 7 and day 14 to determine lineage potential of cultured HSCs (Fig. 6a). HSCs co-cultured with stromal cells/progenitor cells had a higher plating efficiency than HSCs cultured without stroma. HSCs co-cultured with $\mathrm{Sca}{ }^{+}$or $\mathrm{CD}^{+}{ }^{+} 6^{+}$progenitors produced more granulocyte/macrophage (GM) lineages colonies compared with HSCs co-cultured with $\mathrm{CD} 166^{+}$progenitors (Supplementary Fig. 7a). To determine if cultured HSCs could functionally reconstitute multi-lineage hematopoiesis in vivo, we transplanted 200 cultured HSCs with $2 \times 10^{5}$ whole bone marrow cells into lethally irradiated mice. We observed that in vitro co-culture with $\mathrm{Sca}^{+}$and $\mathrm{CD}^{+} 46^{+}$progenitors strongly promoted survival and maintenance of multi-lineage reconstitution by HSC. In contrast, there was little engraftment by HSCs previously cultured without stroma under these minimal culture conditions. Intriguingly, HSCs cultured with $\mathrm{CD} 166^{+}$progenitors had lower myeloid engraftment but similar B- and T-cell engraftment when compared with HSCs cultured with $\mathrm{Sca}^{+}$or $\mathrm{CD}^{+} 46^{+}$ progenitors (Fig. 6b). It is possible that prolonged in vitro culture of the identified progenitors might detrimentally affect their ability to support HSCs, due to in vitro culture-induced apoptosis, senescence, or other changes. To test this possibility, we performed a co-culture experiment using in vitro expanded progenitors instead of freshly isolated ones. We observed a reduced but similar pattern of engraftment (Supplementary Fig. 7b). Our data indicate that these three progenitors were capable of maintaining HSCs and therefore possessed, at least in vitro, functional HSC niche activity.

To test the ability of the mesenchymal stromal progenitors to support HSCs in vivo, we took advantage of the Scal ${ }^{+}$cells' ability to home to the marrow and developed a system for altering the mesenchymal stromal cell population within the HSC niche. We sorted Scal ${ }^{+}$cells from mice expressing the diphtheria toxin receptor (DTR) under control of a tamoxifen-inducible promoter (UBC-creERT2/iDTR) and from matched control (Cre-/iDTR) mice $^{24}$ and injected them into sub-lethally irradiated $\mathrm{C} 57 \mathrm{BL} / \mathrm{Ka}$ mice (Fig. 6c). Under normal conditions, mice are not sensitive to diphtheria toxin. However, when we injected tamoxifen 3 weeks post transplantation, DTR was expressed only in the transplanted cells. Thus, the diphtheria toxin could selectively ablate the injected UBC-creERT2/iDTR Sca1 ${ }^{+}$cells and any cells that were derived from them (Fig. 6c). All three stromal populations were decreased, supporting a model where $\mathrm{CD} 146^{+}$and $\mathrm{CD} 166^{+}$ cells are derived from Sca1 ${ }^{+}$cells (Fig. 6c). We analysed the LSK and LT-HSC populations and found that the total cell number of both was significantly decreased (Fig. 6e). This suggests that the 
a

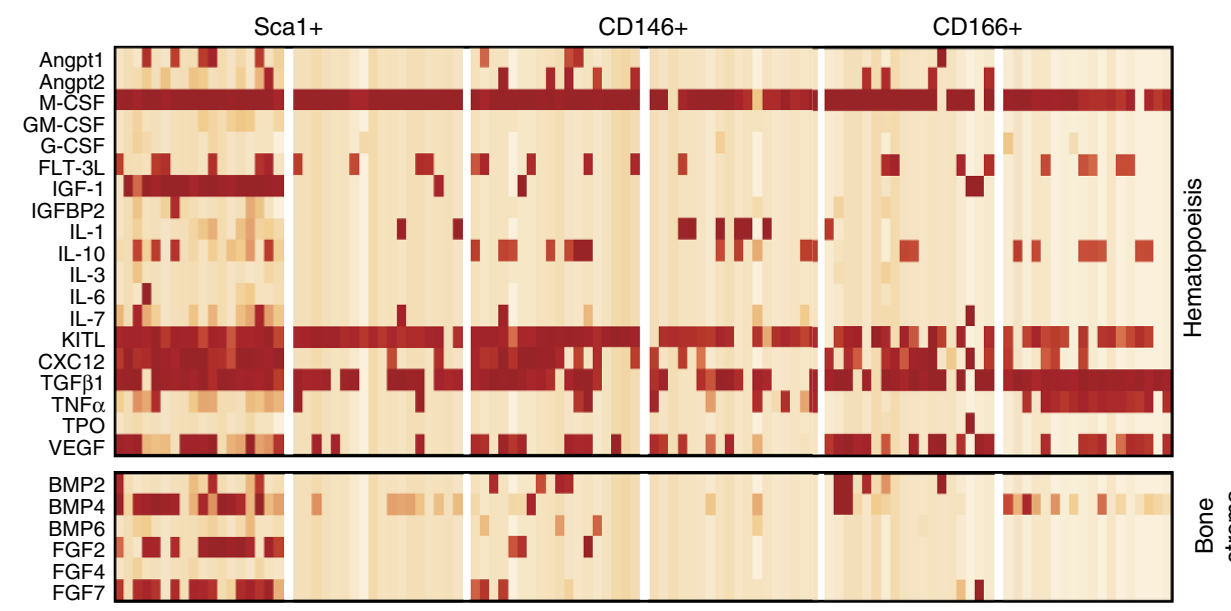

๑)
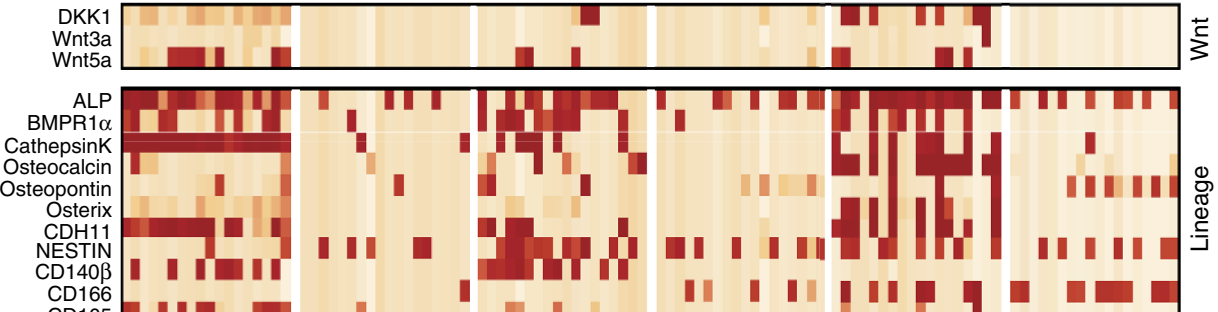

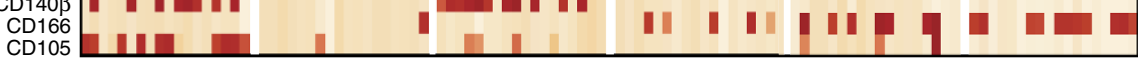
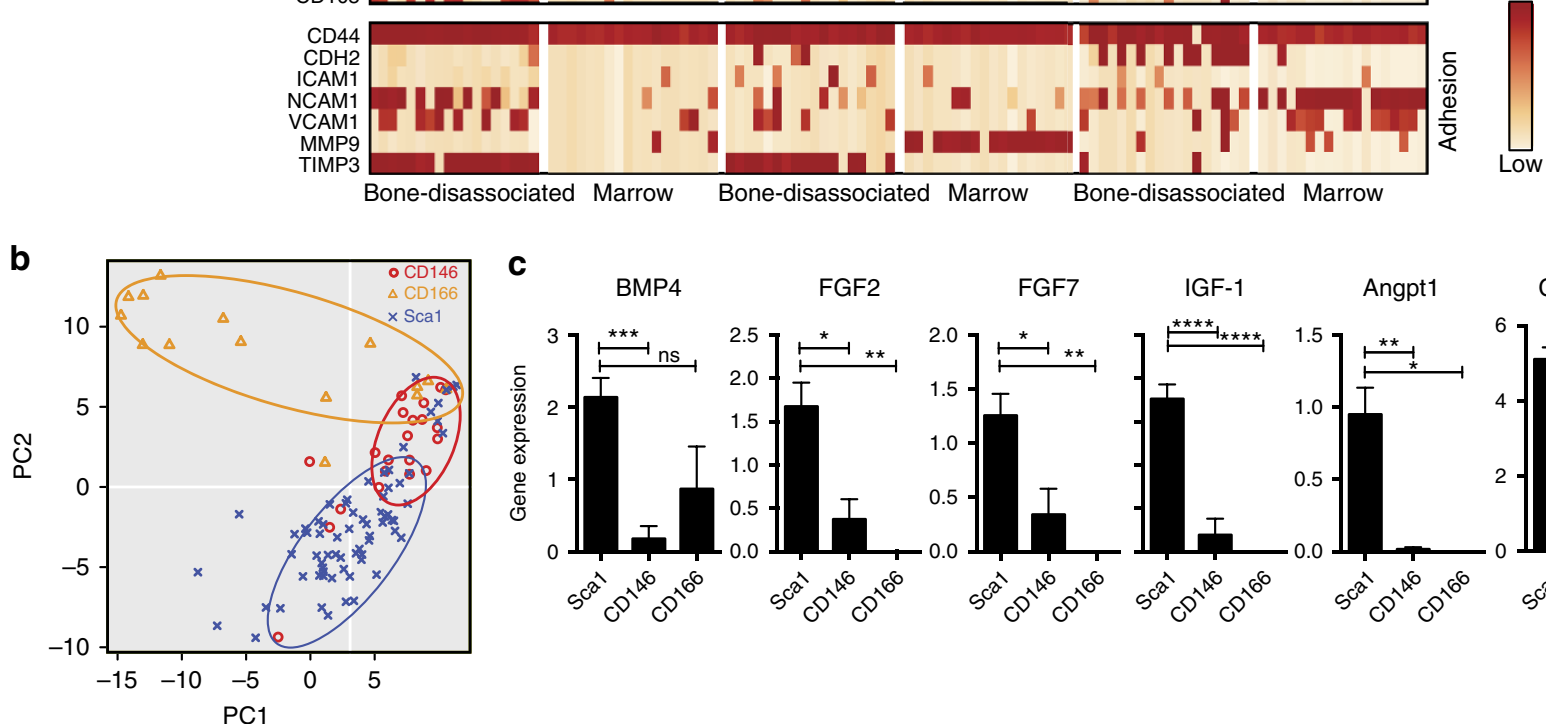

C

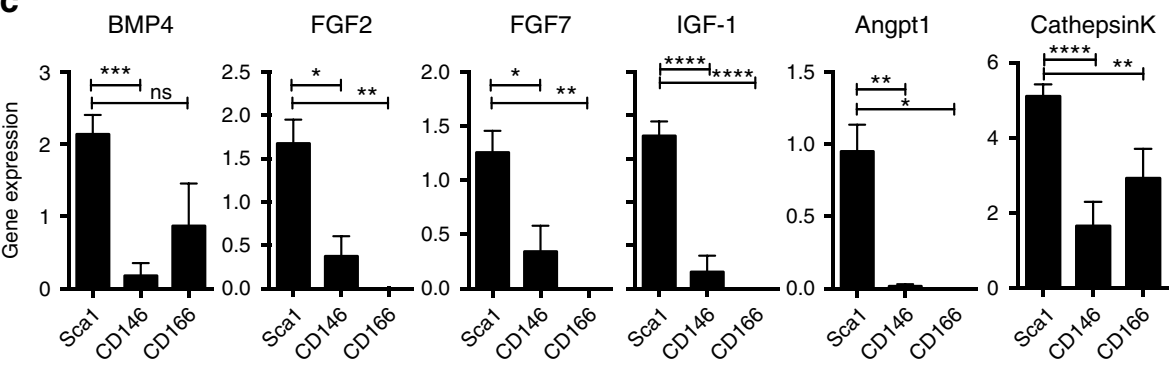

d

Angpt2 Timp3

KitL
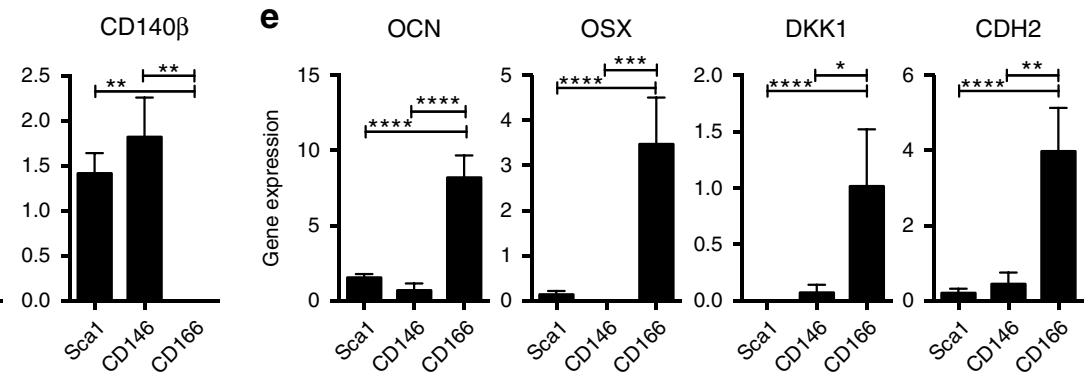

Figure 5 | Bone-disassociated mesenchymal progenitors and marrow stromal cells possess different gene expression profiles. (a) Representative qRT-PCR of 18 single cells from $\mathrm{CD}_{146}{ }^{+}, \mathrm{CD}_{166^{+}}$or Sca1 ${ }^{+}$stromal populations either near the endosteum (bone-disassociated) or in the marrow (Marrow) using the Fluidigm 48.48 dynamic array. Each column within a given population represents expression in an individual cell. (b) Comparison of the first and second prinicpal components from a principal component analysis of single-cell PCR gene expression data from all 47 genes. (c-e) Expression patterns of genes that are significantly overexpressed in mean \pm s.d. (c) Sca1 ${ }^{+}$cells (d) Sca1 ${ }^{+}$cells and/or CD146 ${ }^{+}$cells, or $(\mathbf{e}) \mathrm{CD}_{166}{ }^{+}$cells. Statistical significance was calculated using one-way ANOVA analysis ${ }^{\star} P \leq 0.05,{ }^{\star \star} P \leq 0.005,{ }^{\star \star \star} P \leq 0.00 ; n=72$. ANOVA, analysis of variance. 
a

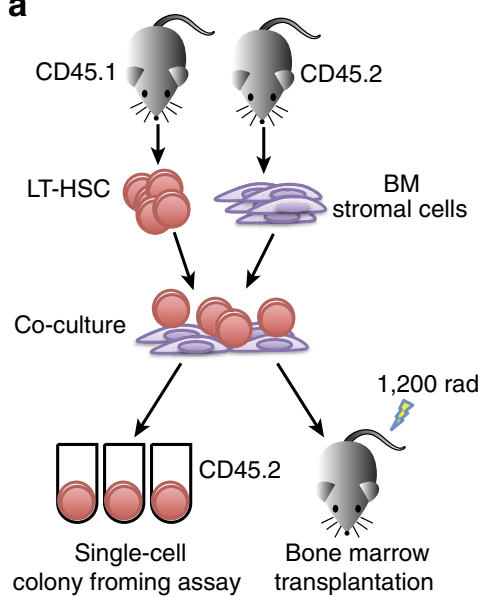

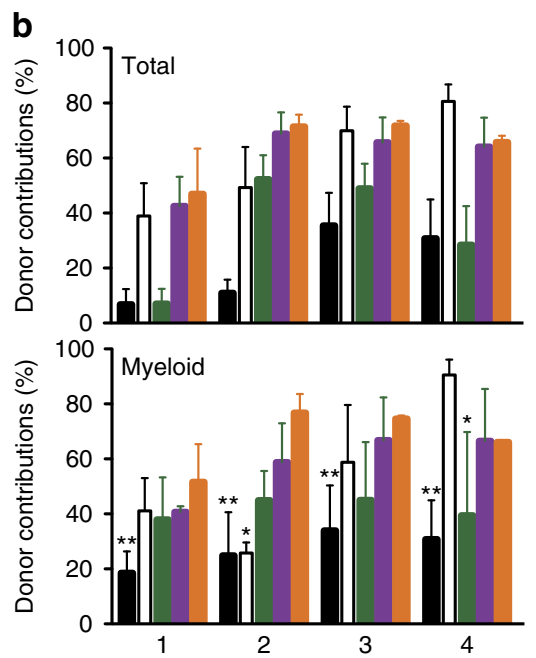

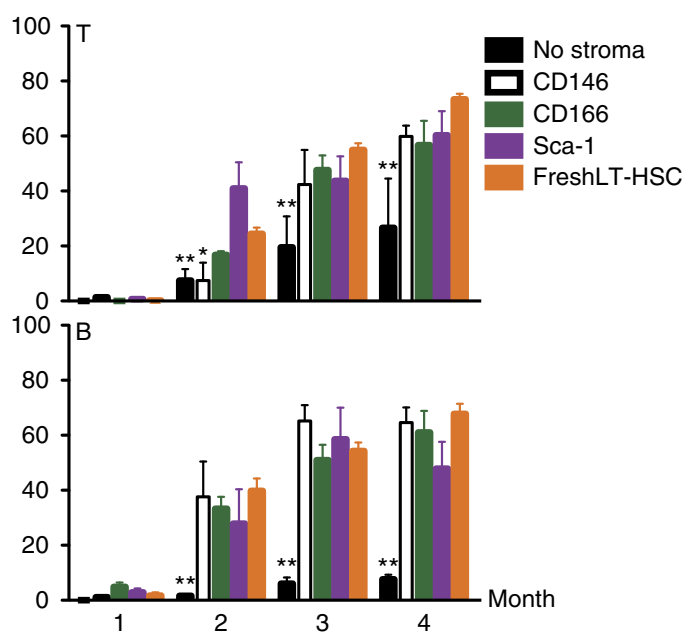

C
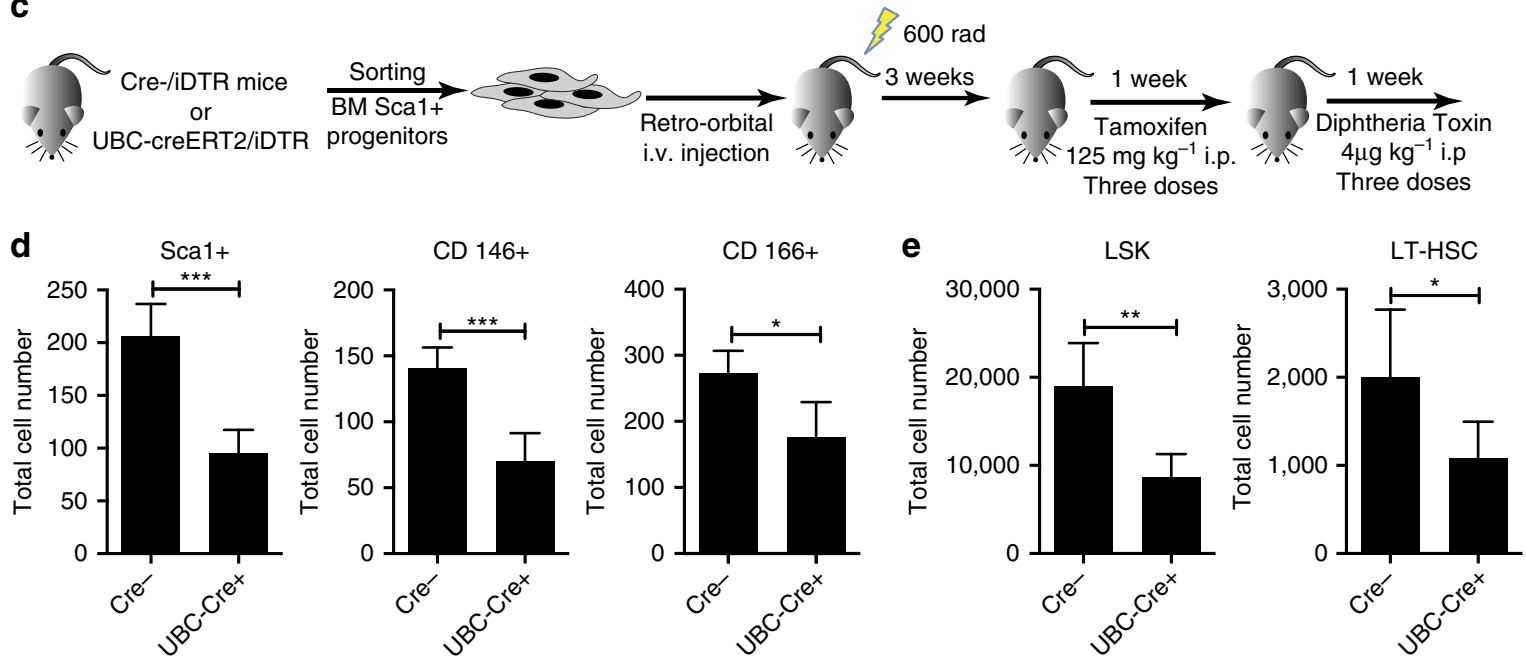

Figure 6 | Bone-disassociated mesenchymal progenitors support HSC maintenance in vitro. (a) LT-HSCs were cultured with mesenchymal progenitors for two days, then analysed for single-cell conlony forming ability or competitive repopulation. (b) Contribution of donor HSCs in the blood assayed 1-4 months after transplantation $(n=8)$. (c) Scal ${ }^{+}$mesenchymal progenitors from $\mathrm{Cre}^{-} /$iDTR or UBC-creERT2/iDTR mice were i.v. injected into mice and then ablated by tamoxifen injection. (d-e) FACS analysis of (d) the mesenchymal stromal progenitor population and (e) the LSK and LT-HSC populations directly after ablation of $\mathrm{Sca}^{+}{ }^{+}$mesenchymal stromal cells $\mathrm{Cre}^{-} n=4$ and $\mathrm{Cre}^{+} n=5$ from two combined experiments. (For $\mathbf{b}$ and $\mathbf{e}$; ${ }^{\star} P \leq 0.05$,

${ }^{\star \star} P \leq 0.005,{ }^{\star \star \star} P \leq 0.001$, mean \pm s.d., student's $t$-test $)$.

mesenchymal stromal progenitor population as a whole is necessary for proper maintenance of HSCs.

KITL in Sca1 ${ }^{+}$cells is important for HSC maintenance. Singlecell qRT-PCR profiling of bone-disassociated progenitors identified several significantly different genes, including TIMP3, CD140 $\beta$, KITL, DKK1, Osterix, CD166, CDH2 and Osteocalcin (Fig. 5c-e and Supplementary Fig. 5d). Altered expression of these genes could contribute to the inferior ability of $\mathrm{CD} 166^{+}$progenitors to support LT-HSC reconstitution, compared with $\mathrm{CD} 146^{+}$and $\mathrm{Sca}^{+}$progenitors. For further analysis, we excluded genes that could represent differences in developmental stages (Osterix and Osteocalcin), or are known surface markers on other cell populations (CD140 $\beta$ and $C D 166)$, or had conflicting reports regarding their role in hematopoiesis $(\mathrm{CDH} 2)$. We chose to further study KITL because of its well-known role in supporting HSCs.

We used a KITL shRNA to knock down the expression of KITL in the $\mathrm{Scal}^{+}$cells. Using the renal sub-capsule assay, we combined fetal skeletal progenitors and Scal ${ }^{+} /$shKITL cells (Fig. 7a), we found that the Scal ${ }^{+} /$shKITL cells still grew in the
BM area of graft (Fig. 7b). However, knockdown of KITL resulted in fewer LSK cells (Fig. 7c) and reduced marrow cellularity (Fig. 7d) in the graft. We conducted qRT-PCR prior to transplantation and droplet digital PCR (ddPCR) post transplantation to demonstrate that the KITL expression was decreased in the KITL shRNA treated cells versus scramble control (Supplementary Fig 8a,b). BM reconstitution assays showed that lethally irradiated mice that received marrow cells from the KITL knockdown graft did not survive, strongly suggesting there were few or no functional HSCs in the marrow of the graft (Fig. 7e). These results suggest that Scal ${ }^{+}$cells are an important source of KITL in the niche needed to maintain and or recruit HSCs.

\section{Discussion}

In this study, we identified a mesenchymal stromal progenitor hierarchy with a primitive mesenchymal stromal progenitor that can give rise to two distinct osteo-progenitors in the adult BM haematopoietic niche. Their identities as niche cells were supported by four independent approaches-in vitro cultures, 
a

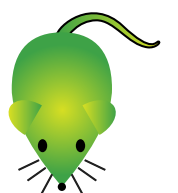

Sorting

Sca1+

stromal progenitors

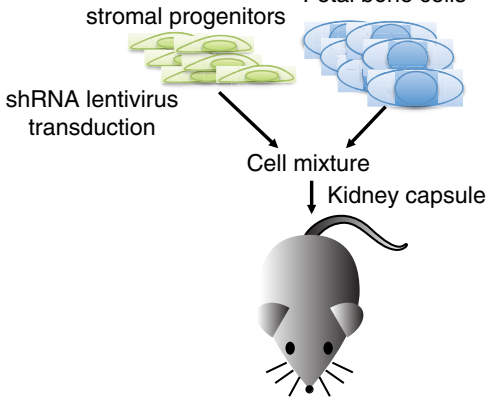

b

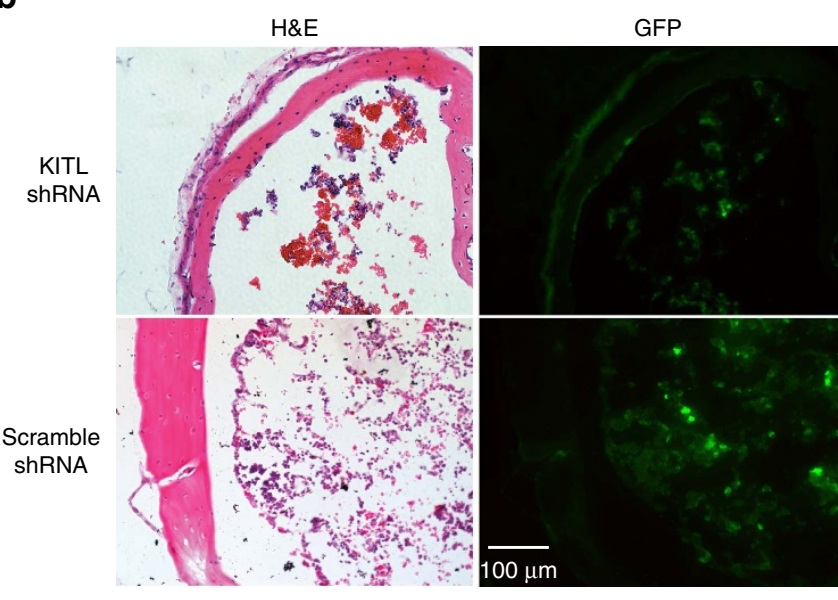

KITL shRNA

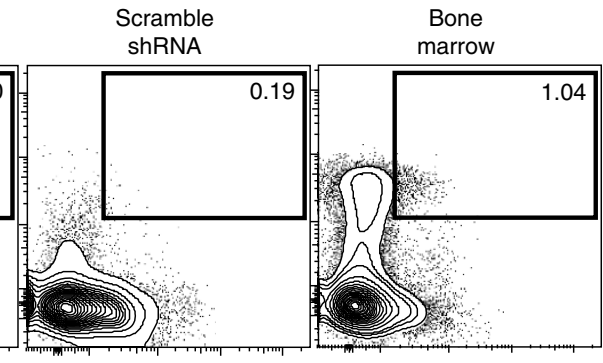

Sca1

d
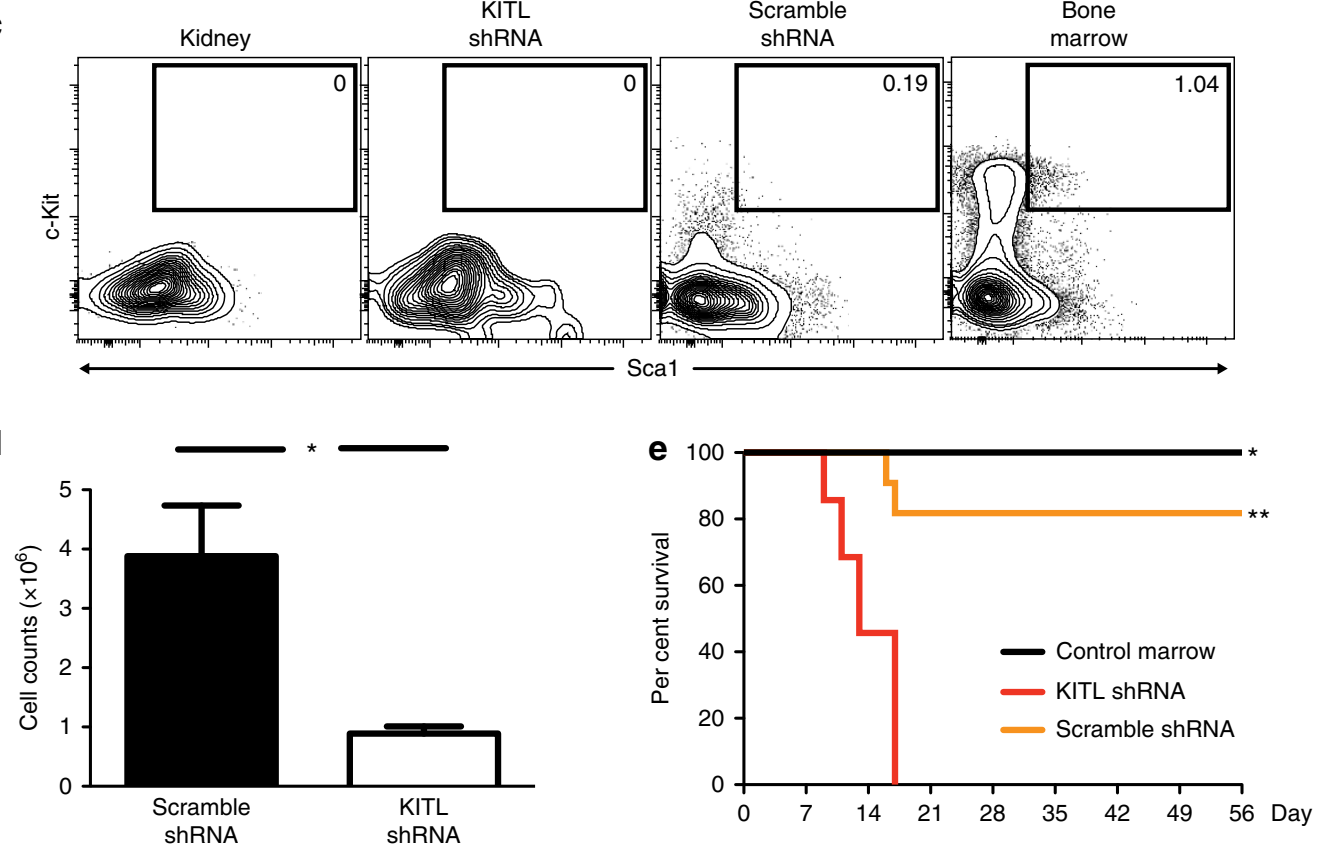

f

CD166+

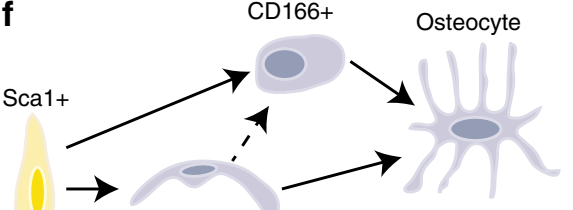

CD146+

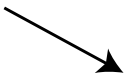

CXCL12 producing stromal cell

Figure 7 | Disruption of KITL production in Sca1 ${ }^{+}$progenitors suppressed hematopoiesis in the ectopic niche. (a) Sca $1^{+}$progenitors were transduced with lentivirus as indicated and 1,000 sorted $\mathrm{GFP}^{+} \mathrm{Sca}^{+}$progenitors were co-transplanted with unmarked fetal skeletal progenitors under the kidney capsule. (b) Representative cross sections of the engrafted ectopic bone stained with H\&E (right) or GFP (left) to identify the donor origin, harvested 1 month after transplant. (c) Representative FACS profiles of LSK frequencies that were pre-gated for live, CD45 ${ }^{+}$lineage ${ }^{-}$cells. (d) Cellularity of the ectopic niche marrow ( ${ }^{\star} P<0.05, n=5$, student's $t$-test). (e) Rescue of lethally irradiated mice with $2 \times 10^{5}$ marrow cells from engrafted ectopic niche generated with either mixed Sca1 ${ }^{+}$/ShKITL progenitors (red) or with Sca1 ${ }^{+} /$Scrambled shRNA progenitors (yellow) or control bone marrow from host tibia (black) $(n=8)$. (f) The schematic summaries our findings such that Sca1 + cells are the most primative giving rise to CXCL12 producing stromal cells, CD146 + cells and CD166 + cells. CD146 + and CD166 + cells are osteo-progenitors. It is possible that CD146 + is an intermidiary cell fate between Sca1 + cells and CD166 + cells. 
in vivo transplantation, in vivo ablation and quantitative single-cell gene expression analyses. Our data indicated that $\mathrm{Sca}{ }^{+}$cells represent common mesenchymal stromal progenitors that can give rise to osteogenic $\mathrm{CD}_{146}{ }^{+}$and $\mathrm{CD}_{166}{ }^{+}$ progenitors and CXCL12-producing stromal cells in the marrow (Fig. 7f). Although the lineage relationship between the CD146 ${ }^{+}$ cells and $\mathrm{CD} 166^{+}$progenitors has not been determined, our gene expression analysis (Fig. 5) suggests that CD146 ${ }^{+}$cells represent a more primitive progenitor than $\mathrm{CD}_{166^{+}}$cells, possibly acting as an intermediary progenitor.

Our finding that $\mathrm{Scal}^{+}$and $\mathrm{CD}^{+} 46^{+}$both localized to perivascular region but have different differentiation potentials in vivo is especially important. It directly supports the notion that not all perivascular stromal cells are identical. Although several perivascular stromal cells have been implicated in HSC maintenance, the lineage relationship among them remains unclear. Leptin receptor is expressed in all three of our mesenchymal progenitors (Supplementary Fig. 6d,e). In contrast, Nestin expression varies among our progenitors. Because of low Nestin expression (Fig. 5a) and detection of very few Scal and nestin co-expressing cells in Nestin-GFP mouse BM (Supplementary Fig. 6a,b), we believe that our Sca1 ${ }^{+}$ progenitors do not overlap with nestin ${ }^{+}$MSCs. All of our mesenchymal progenitors produced CXCL12 (Fig. 5a), a defining characteristic of CAR cells. However, none of the progenitors fits the surface marker definition of CAR cells $\left(\mathrm{CD} 44^{+} \mathrm{CD} 51^{+}\right.$ $\left.\mathrm{CD}_{105}{ }^{+} \mathrm{CD} 140 \mathrm{a}^{+}\right)$. Using an ectopic bone-forming assay and direct intravenous transplantation model, we demonstrated that $\mathrm{Sca}^{+}$progenitors developed into cells that phenotypically resembled CAR cells and produced CXCL12 (Fig. 2f and 4d and Supplementary Fig. 5a,b), suggesting that CAR cells can be derived from the Scal ${ }^{+}$progenitors. More analyses are needed to define the developmental pathway between $\mathrm{Sca}^{+}{ }^{+}$progenitors and CAR cells in adult mice. The periosteum on the outer portion of the bone also expresses Sca1. We isolated the periosteum by scrapping and performed a CFU assay, they failed to produce any colonies and thus are unlikely to have a significant contribution to our population analysis (Supplementary Fig. 2h).

In contrast to fetal $\mathrm{CD}_{105^{+}}{\text {Thy } 1.1^{-}}^{-}$and postnatal progenitors that have the ability to initiate niche formation ${ }^{7,25}$, we found that none of the adult mesenchymal progenitors are capable of niche formation by themselves under comparable conditions, suggesting their ability is limited compared with fetal and postnatal progenitors which can initiate niche formation under minimal conditions. The niche-initiating ability may be suppressed in adult progenitors under homoeostasis conditions to reduce the chances of heterotopic ossification. Production of BMP2, an osteo-inductive cytokine that is involved in heterotopic bone formation, is lower in adult $\mathrm{Scal}^{+}$progenitors compared with fetal osteochondral progenitors (Fig. 5a). However the adult progenitors can participate in generation of niche cells in existing microenvironments and may contribute to the maintenance of the niche. Because $\mathrm{CD} 146^{+}$and $\mathrm{CD} 166^{+}$progenitors form bone after transplantation (Fig. 2b,d), they may be involved in both routine osteocyte turnover and bone regeneration when trauma or injury occurs. Under normal conditions, $\mathrm{Sca}{ }^{+}$progenitors may contribute to the stromal component in the niche, mainly through differentiation into CXCL12-producing stromal cells (Fig. 2f and 3d and Supplementary Fig. 5a). Although Sca1 ${ }^{+}$ progenitors did not form osteocytes immediately after transplantation (Fig. 2b), they eventually contributed to bone formation (Fig. 3f). This process may be accelerated in the event of bone fracture or injury to compensate for the loss of bone progenitors and to guarantee osteo-lineage turnover. Scal knock-out mice have phenotypically normal bones into adulthood. However, over time they exhibit dramatically decreased bone mass that results in brittle bones with increased susceptibility to fractures ${ }^{26}$, suggesting that $\mathrm{Scal}^{+}$progenitors might play a role in bone remodelling.

The HSC niche is far more complex than first reports suggest or than the Drosophila models indicate. The simple model of a niche where a single stromal cell type guides a single type of stem cell in the Drosophila germ line does not fully explain the formation of the mammalian BM niche. The heterogeneity within HSCs and haematopoietic progenitor populations suggests the possible existence of 'micro-niches' within the BM microenvironment. Our detailed analysis of the mesenchymal stromal progenitor hierarchy provides a platform for better understanding the unique properties of each stromal subtype. Although all three mesenchymal progenitors could support multi-lineage hematopoiesis in vitro, their abilities to support myelopoiesis varied (Fig. 6b). LT-HSCs co-cultured with $\mathrm{CD} 66^{+}$cells produced fewer GM colonies (Supplementary Fig. 7a) and lower myeloid output after transplantation (Fig. 6b), indicating $\mathrm{CD} 66^{+}$cells may either preferentially support lymphopoiesis or suppress myelopoiesis. A similar bias was evident in the experiment done by the Suda group using LSK cells co-cultured with $\mathrm{CD} 166^{+} \mathrm{Sca1}{ }^{-}$cells ${ }^{27}$. Recent publications from the Morrison and Link groups suggest the existence of a perivascular niche for HSCs and an endosteal niche for lymphoid progenitors ${ }^{28,29}$. It is interesting to note that Scal ${ }^{+} / \mathrm{CD} 146^{+}$ progenitors localized to the perivascular regions (Fig. 1d,e), whereas $\mathrm{CD} 66^{+}$cells mainly localized to the endosteal surface (Supplementary Fig. 1g). Moreover, canonical Wnt signalling was reported to inhibit lymphopoiesis and non-canonical Wnt signalling facilitated lymphoid commitment ${ }^{30}$. DKK1, a wellknown canonical Wnt signalling inhibitor, is highly expressed in $\mathrm{CD} 66^{+}$cells (Fig. 5e). These data indicate potential mechanisms for how different stromal subsets may influence HSC fate decisions. However, more systematic and mechanistic studies are needed to define how different niche components integrate their activity to provide physiological support for different HSCs and haematopoietic progenitors.

By knocking down KITL expression in transplanted Sca1 ${ }^{+}$ progenitors, we directly demonstrated that $\mathrm{Scal}^{+}$progenitors and their progeny are not merely structural components, but they actively provide functional support to HSCs in the niches (Fig. 7). The impaired ability of KITL knockdown $\mathrm{Scal}^{+}$cells to recruit or support hematopoiesis was reflected in the lower LSK and total cell number harvested from the ectopic niche (Fig. 7c,d). Our data indicate that perivascular $\mathrm{Scal}^{+}$progenitors are an important source of the KITL, consistent with results from the Morrison group ${ }^{23}$. However our results do not exclude the possibility that KITL also plays a role in maintenance and or differentiation of the mesenchymal stromal cell population. Mechanistic studies into the specific role of KITL in the Scal+ stromal cell population are of future interest and would enhance the understanding of the HSC niche. Collectively, our results provide convincing evidence that perivascular $\mathrm{Sca}^{+}$ cells function as a common progenitor of niche components in the murine adult BM.

\section{Methods}

Mice. C57BL/Ka (CD45.2), C57BL/Ka-CD45.1, C57BL/Ka-Thy1.1-CD45.1, C57BL/Ka- Thyl.1-CD45.1-/Tg(CAG-GFP) and B6-Rag-2 ${ }^{-/-} \gamma \mathrm{C}^{-/-}$(CD45.2) mice were maintained by the Animal Resource Center of City of Hope under SPF conditions. Timed embryos from C57BL/Ka-Thy1.1-CD45.1 mice were used in the fetal skeletal progenitors/adult mesenchymal progenitor co-transplantation studies. B6-Rag-2 $2^{-1-} \gamma \mathrm{c}^{-/-}$mice that were $8-12$ weeks old were used as recipients for fetal bone transplantation. C57BL/Ka or C57BL/Ka-CD45.1 mice were used as recipients in HSC and stromal cell transplantation assays. Flk1-GFP Osx1-Cre:GFP, C57BL/6-tdTomato, UBC-CreERT2 and iDTR mice were obtained from Jackson Labs. The Col2.3-GFP mice were a kind gift of Dr Ravi Bhatia. The 
nestin-GFP mice were kindly provided by Dr Qiang $\mathrm{Lu}^{31}$. The CD166-GFP mice ${ }^{32}$ were a generous gift from Dr Ya-Huei Kuo. Mouse care and experimental procedures were performed in accordance with federal guidelines and protocols approved by the Institutional Animal Care and Use Committee at City of Hope.

Isolation of adult mesenchymal progenitors. Tibias and femurs were excised from eight to 12-week-old mice. After cleaning the muscle and connective tissue, the bones were crushed gently with a mortar and pestle in PBS buffer. The crushed mass was washed with PBS to remove all of the BM cells. The remaining bone fragments were incubated at $37^{\circ} \mathrm{C}$ with collagenase $\mathrm{I}\left(3 \mathrm{mg} \mathrm{ml}^{-1}\right.$; Sigma) and gently agitated for $60 \mathrm{~min}$. The digested bones were then filtered through a $40 \mu \mathrm{m}$ strainer (BD Bioscience), pelleted at $200 \mathrm{~g}$ at $4{ }^{\circ} \mathrm{C}$, and resuspended in PBS. The marrow cells were collected and red blood cells were depleted with ACK lysis buffer. The cells were blocked with an anti-CD16/32 antibody and stained with labelled monoclonal antibodies against CD45 (1:100 BioLegend 103128), CD31 (1:100 BioLegend 102418), Ter119 (1:500 BioLegend 116210), Scal (1:20 BioLegend 108133), CD146 (1:25 BioLegend 134705) and CD166 (1:25 R\&D Systems FAB1172P) or antibodies against CD45, CD31, Ter119, CD51(1:100 BioLegend 104105), CD105 (1:100 BioLegend 120410) and Thy1.1 (1:100 BioLegend 140306). The stained cells were then sorted using a FACSAria III sorter (BD Bioscience). The purity of sorted cells was confirmed by flow cytometry to be greater than $99 \%$. All antibodies were purchased from Biolegend or eBioscience unless otherwise noted.

Isolation of LT-HSCs. Haematopoietic stem and progenitor cells were enriched from RBC-lysed BM cells using the c-Kit microbeads kit (Miltenyi Biotec) following the manufacture's instructions. The enriched haematopoietic stem and progenitor cells were then blocked with anti-CD16/32 antibody, stained with labelled antibodies against c-Kit (1:100 BioLegend 105826), Scal (1:100BioLegend 108114), CD150 (1:100 BioLegend 115912), CD48 (1:100 BioLegend 103414), CD135 (1:50 BioLegend 135306) and lineage (CD3 1:100, CD4 1:200, CD8 1:100, CD11b 1:200, B2201:200, Gr-1 1:200, Ter119 1:100 [all Biolegend]), then sorted using a FACSAria III. The LT-HSCs were defined using the following cell-surface markers: lineage-Sca ${ }^{+} \mathrm{c}-\mathrm{Kit}^{+} \mathrm{CD} 150{ }^{+} \mathrm{CD} 48^{-} \mathrm{CD} 135^{-}$.

Stromal cell culture and CFU-F assay. All stromal cells were cultured in MEM-alpha medium (Gibco) containing 20\% fetal bovine serum (FBS; Atlanta Biologicals) at $37^{\circ} \mathrm{C}$ with $5 \% \mathrm{CO}_{2}$. For single-cell CFU-F assays, single stromal cells were sorted using a FACSAria III into 96-well flat bottom plates containing MEM-alpha medium. The colonies were counted after 2 weeks using an inverted microscope (Leica).

LT-HSC and stromal cell in vitro co-culture assay. Bone-disassociated stromal cells $(5,000)$ from CD45.2 mice and CD45.1 ${ }^{+}$LT-HSCs $(1,000)$ were cultured in IMDM medium (Gibco) containing 10\% FBS, $1 \mathrm{ng} \mathrm{ml}^{-1}$ KITL (Peprotech) and $1 \mathrm{ng} \mathrm{ml}^{-1}$ TPO (Peprotech) for 2 days. Harvested CD45.1 ${ }^{+}$HSCs were either sorted individually into 96 -well U bottom plates for the single-cell CFU assay ${ }^{33}$ or transplanted into lethally irradiated ( 1,100 rads) CD 45.2 recipient mice $(250$ cells/ mouse initial seeding HSC number) together with 200,000 CD45.2 ${ }^{+}$helper marrow cells. For the CFU assay the single cells were cultured in IMDM medium (Gibco) containing $10 \% \mathrm{FBS}, 10 \mathrm{ng} \mathrm{m}^{-1}$ of the following cytokines (Peprotech) IL-1a, IL-3, IL-5, IL7, IL-9, IL-10, IL-11, GM-CSF, TPO, EPO, SCF and Flt3, colonies were counted 7 days after seeding. For the transplantation assay. For the transplantation assay, peripheral blood was drawn at the indicated time points and the contribution of donor-derived cells was analysed monthly by flow cytometry.

Transplantation of adult mesenchymal progenitors. The kidney capsule transplantation procedure was performed as follows: 5,000 sorted adult stromal cells alone or with 30,000 unsorted fetal bone cells were mixed with $5 \mu \mathrm{l}$ of matrigel and injected underneath the renal capsule of 8-12-week-old B6-Rag-2-1- $\gamma^{-1}{ }_{-}^{-}$ mice. The grafts were harvested 1 month later and imaged using a Leica M205 FA stereomicroscope. Alternatively, 5,000 sorted adult stromal cells in $100 \mu \mathrm{l}$ PBS were injected intravenously into irradiated 8-12-week-old mice (600 rad). Long bones of recipient mice were harvested 1 month after transplantation.

Tissue section and immunofluorescence staining. Freshly dissected long bones and kidney grafts were fixed in $4 \%$ paraformaldehyde for $30 \mathrm{~min}$ at $4{ }^{\circ} \mathrm{C}$ then decalcified in 10\% EDTA at $4^{\circ} \mathrm{C}$ for 5-7 days. The bones were then embedded in $10 \%$ gelatin in PBS, frozen on dry ice and stored at $-80^{\circ} \mathrm{C}$. Bones were sectioned using the CryoJane taping system (Instrumedics). Sections were blocked with $1 \%$ $\mathrm{BSA}$, then probed with primary antibody at $4{ }^{\circ} \mathrm{C}$ overnight; purified rat-anti-Scal (1:50 BioLegend 108102), purified rat-anti-CD146 (1:50, BioLegend 134702), CD166-Fitc (1:25 R\&D Systems FAB1172F), CD45-Alexa647 (1:50 BioLegend 103124) and/or rabbit-anti-laminin (1:200 Sigma-Aldrich L9393-.2ML). After washing with PBS, the sections were probed with Alexa dye-conjugated antibodies, Invitrogen goat anti-rat-555 or anti-rat-488 and anti-rabbit-555 (Alexa antibodies used at 1:100), then washed and stained with Dapi and mounted in ProLong Gold anti-fade mounting media. Sections were analysed using an Olympus IBX81 microscope. Slides that passed this initial inspection were scanned using a Zeiss LSM 700 confocal microscope or iCys Research imaging cytometer (iCys, CompuCyte). Zen 2012 and Amira 3D Projection and Analysis software were used to create the maximum intensity projections and three-dimensional surface renderings of confocal $z$-stacks, respectively.

Single-cell multi-gene quantitative real-time PCR. We double sorted individual stromal cells into $10 \mu$ l SuperScript III One-Step RT-PCR System with Platinum Taq DNA polymerase (Invitrogen) pre-amplification mixture made with the TaqMAN Gene expression assays in Supplementary Table 1, then reverse transcribed and amplified under the following parameters $15 \mathrm{~min}$ at $50^{\circ} \mathrm{C}$, $\left(2 \min 95^{\circ} \mathrm{C}, 4 \mathrm{~min} 60^{\circ} \mathrm{C}\right.$ ) for 22 cycles, $15 \mathrm{~min}$ at $4{ }^{\circ} \mathrm{C}$. The resulting amplified cDNAs were diluted 1:4 and analysed by Q-PCR using the Fluidigm Biomark Dynamic Array following the manufacturer's instructions. The data were processed using the Biomark system software suite and analysed/normalized using the Fluidigm SINGuLAR Toolset 3.0. Outliers were determined using the built-in outlier identification function within the SINGuLAR Toolset and principal component analysis. The TaqMan Gene Expression Assays (Applied Biosystems) assays used are listed in Supplementary Table 1.

Quantitative real-time PCR. RNA was extracted from sorted cells using an RNeasy RNA isolation kit (Qiagen) then reverse transcribed into CDNA with the Sensiscript RT kit (Qiagen). The cDNA was amplified for specific targets using TaqMan in a ViiA 7 Real-Time PCR System (Life Technologies). The thermal cycling parameters were 40 cycles of $95^{\circ} \mathrm{C}$ for $15 \mathrm{~s}$ and $60^{\circ} \mathrm{C}$ for $1 \mathrm{~min}$. Data were analysed with the 7500 Fast System SDS software. The TaqMan assays used are listed in Supplementary Table 1.

Droplet Digital PCR (ddPCR). Sorted GFP $+/$ - cells from kidney grafts were collected for RNA isolation and cDNA synthesis, as above. An amount of $1 \mathrm{ul}$ cDNA was mixed with $2 \times$ concentrated ddPCR Supermix for Probes (Cat. 186-3026, BioRad) and Taqman probe for KITL and GAPDH (Applied Biosystems) according to manufacturer's protocol. Droplets were generated with $20 \mu \mathrm{l}$ PCR reaction mix and $70 \mu \mathrm{l}$ oil in a DG8 cartridge using QX 200 droplet generator (Bio-Rad). The resulting droplets were then transferred into a PCR plate. After PCR amplification, the plate was read using a QX200 droplet reader to provide absolute quantification of the target genes. QuantaSoft software (Bio-Rad) was used to analyse the positive counts per sample. The counts of target genes (copies/ul reaction) for each sample were normalized to internal GAPDH control.

shRNA transduction. KITL-specific shRNA knockdown constructs ${ }^{7}$ using pll3.7 shRNA cloning vector and active lentiviral stocks were generated as previously described $^{34}$, using the VSV.G and CMVDR8.74 packaging plasmids along with jetPRIME transfection reagent (manufactures protocol). The sorted, adult bonedisassociated $\mathrm{Scal}^{+}$cells were resuspended in MEM-alpha medium with $15 \%$ fetal calf serum (FCS) and transduced with shRNA lentiviral vectors carrying the KITLspecific shRNA or a scrambled control. Forty-eight hours after transduction, 1,000 $\mathrm{GFP}^{+}$cells were sorted and transplanted under the kidney capsule with 20,000 fetal skeletal progenitors in $5 \mu \mathrm{l}$ matrigel. The grafts were harvested 1 month later for analysis.

Statistical Analysis. All data are presented as mean \pm s.d. and two group comparisons were performed using a two-tailed unpaired Student's $t$-test. Analysis of variance analysis was conducted on the single-cell qRT-PCR gene expression data, while only 18 cells are shown on the heatmap they are representative of several repeats that amounted to 72 individual cells per group and were used for the one-way analysis of variance analysis. Survival was measured using Kaplan-Meier analysis. A value of $P \leq 0.05$ was considered statistically significant for all tests. Statistical test were not used to determine the sample size. No randomization was used to allocate animals to particular groups; age and sex-matched recipients were used for transplantation experiments. The investigators were not blinded to experimental groups during analysis. Statistical analyses were performed using GraphPad Prism software v.6.

Data Availability. The authors declare that all data supporting the findings of this study are available within the article and its Supplementary Information files or from the corresponding author upon reasonable request.

\section{References}

1. Morrison, S. J. \& Spradling, A. C. Stem cells and niches: mechanisms that promote stem cell maintenance throughout life. Cell 132, 598-611 (2008).

2. Moore, K. A. \& Lemischka, I. R. Stem cells and their niches. Science 311, 1880-1885 (2006).

3. Ehninger, A. \& Trumpp, A. The bone marrow stem cell niche grows up: mesenchymal stem cells and macrophages move. J. Exp. Med. 208, 421-428 (2011). 
4. Morrison, S. J. \& Scadden, D. T. The bone marrow niche for haematopoietic stem cells. Nature 505, 327-334 (2014).

5. Karp, J. M. \& Leng Teo, G. S. Mesenchymal stem cell homing: the devil is in the details. Cell Stem Cell 4, 206-216 (2009).

6. da Silva Meirelles, L., Caplan, A. I. \& Nardi, N. B. In search of the in vivo identity of mesenchymal stem cells. Stem Cells 26, 2287-2299 (2008).

7. Chan, C. K. F. et al. Endochondral ossification is required for haematopoietic stem-cell niche formation. Nature 457, 490-494 (2009).

8. Zhou, B. O., Yue, R., Murphy, M. M., Peyer, J. G. \& Morrison, S. J. Leptin-receptor-expressing mesenchymal stromal cells represent the main source of bone formed by adult bone marrow. Cell Stem Cell 15, 154-168 (2014).

9. Guezguez, B. et al. Regional localization within the bone marrow influences the functional capacity of human HSCs. Cell Stem Cell 13, 175-189 (2013).

10. Kunisaki, Y. et al. Arteriolar niches maintain haematopoietic stem cell quiescence. Nature 502, 637-643 (2013).

11. Sacchetti, B. et al. Self-renewing osteoprogenitors in bone marrow sinusoids can organize a hematopoietic microenvironment. Cell 131, 324-336 (2007).

12. Ohneda, O. et al. ALCAM (CD166): its role in hematopoietic and endothelial development. Blood 98, 2134-2142 (2001).

13. Yousif, L. F., Di Russo, J. \& Sorokin, L. Laminin isoforms in endothelial and perivascular basement membranes. Cell Adh. Migr. 7, 101-110 (2013).

14. Omatsu, Y. et al. The essential functions of adipo-osteogenic progenitors as the hematopoietic stem and progenitor cell niche. Immunity 33, 387-399 (2010).

15. Kalajzic, I. et al. Use of type I collagen green fluorescent protein transgenes to identify subpopulations of cells at different stages of the osteoblast lineage. J Bone Miner Res 17, 15-25 (2002).

16. Rodda, S. J. \& McMahon, A. P. Distinct roles for Hedgehog and canonical Wnt signaling in specification, differentiation and maintenance of osteoblast progenitors. Development 133, 3231-3244 (2006).

17. Sugiyama, T., Kohara, H., Noda, M. \& Nagasawa, T. Maintenance of the hematopoietic stem cell pool by CXCL12-CXCR4 chemokine signaling in bone marrow stromal cell niches. Immunity 25, 977-988 (2006).

18. Costa, A. G., Cusano, N. E., Silva, B. C., Cremers, S. \& Bilezikian, J. P. Cathepsin K: its skeletal actions and role as a therapeutic target in osteoporosis. Nat. Rev. Rheumatol. 7, 447-456 (2011).

19. Nakajima, H. et al. TIMP-3 recruits quiescent hematopoietic stem cells into active cell cycle and expands multipotent progenitor pool. Blood 116, 4474-4482 (2010).

20. Armulik, A., Abramsson, A. \& Betsholtz, C. Endothelial/pericyte interactions. Circ. Res. 97, 512-523 (2005)

21. Walsh, N. C. \& Gravallese, E. M. Bone remodeling in rheumatic disease: a question of balance. Immunol. Rev. 233, 301-312 (2010).

22. Méndez-Ferrer, S. et al. Mesenchymal and haematopoietic stem cells form a unique bone marrow niche. Nature 466, 829-834 (2010).

23. Ding, L., Saunders, T. L., Enikolopov, G. \& Morrison, S. J. Endothelial and perivascular cells maintain haematopoietic stem cells. Nature 481, 457-462 (2012).

24. Buch, T. et al. A Cre-inducible diphtheria toxin receptor mediates cell lineage ablation after toxin administration. Nat. Methods 2, 419-426 (2005).

25. Chan, C. K. et al. Identification and specification of the mouse skeletal stem cell. Cell 160, 285-298 (2015).

26. Bonyadi, M. et al. Mesenchymal progenitor self-renewal deficiency leads to age-dependent osteoporosis in Sca-1/Ly-6A null mice. Proc. Natl Acad. Sci. USA 100, 5840-5845 (2003).

27. Nakamura, Y. et al. Isolation and characterization of endosteal niche cell populations that regulate hematopoietic stem cells. Blood 116, 1422-1432 (2010).

28. Ding, L. \& Morrison, S. J. Haematopoietic stem cells and early lymphoid progenitors occupy distinct bone marrow niches. Nature 495, 231-235 (2013).
29. Greenbaum, A. et al. CXCL12 in early mesenchymal progenitors is required for haematopoietic stem-cell maintenance. Nature 495, 227-230 (2013).

30. Malhotra, S. et al. Contrasting responses of lymphoid progenitors to canonical and noncanonical Wnt signals. J Immunol 181, 3955-3964 (2008).

31. Wang, J. et al. Transcriptome analysis of neural progenitor cells by a genetic dual reporter strategy. Stem Cells 29, 1589-1600 (2011).

32. Weiner, J. A. et al. Axon fasciculation defects and retinal dysplasias in mice lacking the immunoglobulin superfamily adhesion molecule BEN/ALCAM/ SC1. Mol. Cell. Neurosci. 27, 59-69 (2004).

33. Franco, C. B., Chen, C.-C., Drukker, M., Weissman, I. L. \& Galli, S. J. Distinguishing mast cell and granulocyte differentiation at the single-cell level. Cell Stem Cell 6, 361-368 (2010).

34. Metz, M. et al. Mast cells can enhance resistance to snake and honeybee venoms. Science (New York, NY) 313, 526-530 (2006).

\section{Acknowledgements}

We thank Drs Margaret Morgan, David L. DiGiusto, Nancy Linford and Ravi Bhatia for advice and critical reading of the manuscript. We thank Donna Isbell and Danielle Kim for animal care. This study was supported in part by grants to C.-C.C from the Children Leukemia Research Association, the ThinkCure! Foundation, the Margaret E. Early Medical Research Trust, the Tim Nesvig Lymphoma Research Fund, the American Cancer Society Grant 128766-RSG-15-162 and the National Institute of Health (R00 HL087936). X.H. was supported by a California Institute for Regenrative Medicine (CIRM) training grant. Research reported in this publication included work performed in the Analytical Cytometry Core and Animal Resource Center supported by the National Cancer Institute of the National Institutes of Health under award number P30CA33572. The content is solely the responsibility of the authors and does not necessarily represent the official views of the National Institutes of Health.

\section{Author contributions}

X.H., M.G., L.W., X.J., J.L.M. and B.K. conducted the experiments, X.H., M.G., L.W. and C.-C.C. designed the experiments. C.D.W. contributed to the single-cell qRT-PCR data analysis. I.T. helped slide scanning using the iCys Research imaging cytometer. X.H M.G. and C.-C.C. wrote the paper.

\section{Additional information}

Supplementary Information accompanies this paper at http://www.nature.com/ naturecommunications

Competing financial interests: The authors declare no competing financial interests.

Reprints and permission information is available online at http://npg.nature.com/ reprintsandpermissions/

How to cite this article: $\mathrm{Hu}, \mathrm{X}$. et al. Identification of a common mesenchymal stromal progenitor for the adult haematopoietic niche. Nat. Commun. 7, 13095 doi: 10.1038/ncomms13095 (2016)

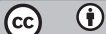

This work is licensed under a Creative Commons Attribution 4.0 International License. The images or other third party material in this article are included in the article's Creative Commons license, unless indicated otherwise in the credit line; if the material is not included under the Creative Commons license, users will need to obtain permission from the license holder to reproduce the material To view a copy of this license, visit http://creativecommons.org/licenses/by/4.0/

C) The Author(s) 2016 